\title{
Computing Edge Weights of Symmetric Classes of Networks
}

\author{
Hafiz Usman Afzal, ${ }^{1}$ Muhammad Javaid $\mathbb{D}^{1},{ }^{1}$ Abdulaziz Mohammed Alanazi, \\ and Maryam Gharamah Alshehri ${ }^{2}$ \\ ${ }^{1}$ Department of Mathematics, School of Science, University of Management and Technology, Lahore 54770, Pakistan \\ ${ }^{2}$ Department of Mathematics, University of Tabuk, Tabuk, Saudi Arabia
}

Correspondence should be addressed to Muhammad Javaid; javaidmath@gmail.com

Received 22 January 2021; Revised 17 February 2021; Accepted 1 March 2021; Published 20 March 2021

Academic Editor: Ali Ahmad

Copyright ( $2021 \mathrm{Hafiz}$ Usman Afzal et al. This is an open access article distributed under the Creative Commons Attribution License, which permits unrestricted use, distribution, and reproduction in any medium, provided the original work is properly cited.

\begin{abstract}
Accessibility, robustness, and connectivity are the salient structural properties of networks. The labelling of networks with numeric numbers using the parameters of edge or vertex weights plays an eminent role in the study of the aforesaid properties. The systems interlinked in a network are transformed into a graphical network, and specific numeric labels assigned to the converted network under certain rules assist us in the regulation of data traffic, bandwidth, and coding/ decoding of signals. Two major classes of such network labellings are magic and antimagic. The notion of super $(a, 0)$ edgeantimagic labelling on networks was identified in the late nineties. The present article addresses super $(a, 0)$ edge antimagicness of union of the networks' star $S_{n}$, the path $P_{n}$, and copies of paths and the rooted product of cycle $C_{n}$ with $K_{2, m}$. We also provide super $(a, 0)$ edge-antimagic labelling of the rooted product of cycle $C_{n}$ and planar pancyclic networks. Further, we design a super $(a, 0)$ edge-antimagic labelling on a pancyclic network containing chains of $C_{6}$ and three different symmetrically designed lattices. Moreover, our findings have also been recapitulated in the shape of 3-D plots and tables.
\end{abstract}

\section{Introduction}

In this section, we shall define our problem and explain the objective of this study in Section 1.1, followed by Section 1.2, consisting of the definitions and results which we will use in our findings. Some previously performed work in this area will also be discussed in this section. Moreover, Section 1.3 concerns with applications of antimagic and magic labelling in various branches of networking, engineering, and computer science.

1.1. Problem Definition and Objective of the Study. In the fields of networking and computer science, the magic and antimagic labelling on networks are designed due to their extensive applications. Numerous results have been obtained on numeric labelling of several operations on networks such as Cartesian, lexicographical, corona, and modular products of various kinds of connected networks (see $[1,2]$ for instance). The present article addresses super $(a, 0)$ edge-antimagic labelling of the rooted product of $K_{2, m}$ and $C_{n}$ taking its disjoint union with the star, path, and copies of paths. We shall also design super $(a, 0)$ edgeantimagic labelling on rooted product of specifically designed planar pancyclic networks with cycle $C_{n}$. Moreover, we shall design super $(a, 0)$ edge-antimagic labelling on planar pancyclic networks containing chains of $C_{6}$ and three different symmetric lattice networks (notated as $\mathbb{L}_{n}^{1}, \mathbb{L}_{n}^{2}$, and $\mathbb{L}_{n}^{3}$ ). Except lattice networks, interestingly, all networks discussed in this note are planar. The overlapping probability of various networking elements minimizes in the course of planar networks. In organizations, this issue of entities' overlapping is one of the major reasons of inefficiency. The test ready antimagic labellings obtained in this note on particular networks can be utilized in various projects of computer science and engineering admitting suitable and equivalently designed schemes of networking. 
1.2. Definitions and Preliminaries. Some useful definitions and preliminary results in the context of this note shall be discussed in this section. We will also mention some relevant studies previously done in this field.

An ordered 2-tuple comprising two sets, i.e., set of nodes termed as vertex set $V(\mathbb{G})$ and connection between these vertices termed as edge set $E(\mathbb{G})$, is called a network $\mathbb{G}$, where $E(\mathbb{G})$ is contained in $V(\mathbb{G}) \times V(\mathbb{G})$. A network $\mathbb{G}$ can either be connected or consists of connected components. We will consider nonempty and simple networks throughout, having $V(\mathbb{G})$ as its vertex set and $E(\mathbb{G})$ as its edge set with order $|V(\mathbb{G})|=p$ and size $|E(\mathbb{G})|=q$. The network $\mathbb{G}$ in this case is termed as a $(p, q)$-network. Reference [3] is referred to for further discernment into the network terminologies.

A labelling is a function that maps +ive integers (nonzero) onto the component(s) of a network under specified constraints. If the components include vertices and edges both, then this labelling is termed as total. The labellings are referred to as vertex or edge labellings if they cover, respectively, $V(\mathbb{G})$ or $E(\mathbb{G})$ alone in the domain. Magic and antimagic labelling are two main classes of labelling. Precisely, equal or unequal vertex/edge weights refer to magic or antimagic labellings, respectively.

Definition 1. For a $(p, q)$-network $\mathbb{G}=(V(\mathbb{G}), E(\mathbb{G}))$, the bijective function $\delta$ form $V(\mathbb{G}) \cup E(\mathbb{G})$ onto $\{1,2, \ldots, p+q\}$ is termed as $(a, d)$ edge-antimagic total labelling with the constraint that the edge weights $\delta(x)+\delta(x y)+\delta(y)$, for each $x y \in E(\mathbb{G})$, constitute a sequence of consecutive positive integers, where $a$ is the minimum edge weight and common difference is $d . \mathbb{G}$ is referred to be an $(a, d)$ edge-antimagic total network, if such a labelling exists.

Definition 2. A super $(a, d)$ edge-antimagic total labelling is in fact an $(a, d)$ edge-antimagic total labelling in which minimum labels $1,2, \ldots, p$ are assigned to vertices of the $(p, q)$-network $\mathbb{G}$. $\mathbb{G}$ is termed to be a super $(a, d)$ edgeantimagic total network in this case.

In Definitions 1 and 2, the minimum edge weight $a$ becomes constant $c$ at $d=0$, for all edges $x y \in E(\mathbb{G})$, which is referred to as magic sum or magic constant for the network $\mathbb{G}$.

Definition 3. A network $\mathbb{G}$ is termed as a pancyclic network if it contains cycle of every order from 3 to $|V(\mathbb{G})|$.

Definition 4. Let $\mathbb{G}_{1}$ and $\mathbb{G}_{2}$ be two simple networks. The network obtained by taking $\left|V\left(\mathbb{G}_{1}\right)\right|$ copies of $\mathbb{G}_{2}$ and then for each point (vertex) $v_{j}$ in $\mathrm{V}$ (called the root vertex) $\left(G_{1}\right), v_{j}$ is being replaced with the $j_{\text {th }}$ copy of $\mathbb{G}_{2}$, termed as the rooted product of the networks $\mathbb{G}_{1}$ and $\mathbb{G}_{2}$. It is notated as $\mathbb{G}_{1}^{\circ} \mathbb{G}_{2}$.

Further in the article, the abbreviations are being used as given in. Table 1.

We further provide some specific definitions within the corresponding section of our main results' section.

The idea of magic labelling on networks was identified by Sadlácek in 1963 [4]. The notion of antimagic labelling, for vertex sums of networks, was presented by Ringel and
TABLE 1: Notations and their abbreviations used in the article.

\begin{tabular}{lc}
\hline Terminology & Abbreviation \\
\hline$(a, d)$ edge-antimagic & $(a, d)$-EAM \\
Super $(a, d)$ edge-antimagic & S- $(a, d)$-EAM \\
\hline
\end{tabular}

Hartsfield [5] later. Kotzig and Rosa brought into the light the concept of magic valuations of networks in [6] which was in fact the $(a, 0)$-EAM total labelling on networks (studied by Ringel and Llado [7] in 1996). The notion of S- $(a, 0)-$ EAM total labelling of networks was defined by Enomoto et al. [8] with the terminology super edge-magic labelling. Simanjantuk et al. highlighted $(a, d)$-EAM total labelling of networks in [9] in year 2000.

The literature of $(a, 0)$-EAM total labelling of networks includes the following interesting and useful conjectures.

Conjecture 1. All trees admit (a,0)-EAM total labelling [6].

Conjecture 2. All trees admit $S$ - $(a, 0)-E A M$ total labelling [8].

Graph theorists, in the support of Conjecture 2, have been rectifying several particular classes of trees. Using an encryption of a computer programme, this conjecture has been verified for the trees having at most 17 vertices by Lee and Shah [10]. Specifically, the derivations can be seen for stars, subdivided stars $[11,12,13,14,15], w$-trees $[16,17,18]$, banana trees [19], caterpillars [20], subdivided caterpillars [21], and the union of books and stars [22]. Further relevant works can be found in [23-25]. However, this conjecture is still open for working. Enomoto et al. proved that if a simple $(p, q)$-network $\mathbb{G}$ is $\mathrm{S}$ - $(a, 0)$-EAM total, then $2 p-3$ is at least $q$ [8]. They further derived that the network $K_{m, n}$ is $\mathrm{S}$ - $(a, 0)$-EAM total $\Leftrightarrow m$ or $n$ is 1 . Figueroa-Centeno et al. derived that the union of networks $K_{1, m} \cup K_{1, n}$ is S- $(a, 0)$ EAM total if either $m=\eta_{1}(n+1)$ or $n=\eta_{2}(n+1)$ [26]. The network $C_{n}$ is also proved to be S- $(a, 0)$-EAM total only if $n \equiv 1(\bmod 2)$ in [8]. In [27], $C_{3} \cup C_{n}$ has been proven to be S- $(a, 0)$-EAM total only when $6 \leq n \equiv 0(\bmod 2)$. The generalized prism $D_{m, n}$ is proven to be S- $(a, 0)$-EAM total for all odd values of $m$ in [28]. Baig et al. classified a class of planar pancyclic networks in [29] and exhibited its S- $(a, 0)$-EAM total labelling for all possible values of the parameters involved. An immensely advantageous lemma on S- $(a, 0)$ EAM total networks is as follows. Liu at al. studied the bounds of the minimum and maximum edge weights for super $(a, d)$ - EAM total labelling on a generalized class of subdivided caterpillars in [30] for various values of $d$. In [31], Ahmad et al. studied the super $(a, 0)$-EAM total labelling of certain Toeplitz graphs combined with isolated vertices $n K_{1}$, for various values of $n$ (also known as super edge-magic deficiency of networks). The properties and existence of super $(a, d)$ vertex-antimagic labelling of regular graphs have been discussed in [32]. In [33], Ahmad et al. constructed the $\alpha$ - labelling, a special case of graceful labelling (labelling in which distinct edge weights are considered with respect to the difference of vertices' labels) on trees, and transformed this labelling to edge-antimagic vertex labelling 
of trees. In [34], S- $(a, 0)$-EAM total labelling on the graphs $G \cup n K_{1}$ has been studied, where $G$ represents the unicyclic graph, whereas S- $(a, 0)$-EAM total labelling of networks like zig-zag triangle and disjoint union of combs and stars has been studied in [35].

Lemma 1 (see [28]). A ( $\wp, \mathfrak{\Im})$-network $\mathbb{G}$ is $S$ - $(a, 0)$-EAM total if and only if there is a bijection $\delta: V(\mathbb{G}) \longrightarrow\{1,2$, $\ldots, \wp\}$ such that the set $S=\{\delta(x)+\delta(y) \mid x y \in E(\mathbb{G})\}$ consists of $\mathfrak{\Im}$ consecutive integers. In such a case, $\delta$ extends to an $S$ - $(a, 0)-E A M$ total labelling of $\mathbb{G}$ with magic constant $\mathfrak{R}=$ $\wp+\mathfrak{I}+s$, where $s=\min (S)$ and $S=\{\mathfrak{R}-(\wp+1), \mathfrak{R}-(\wp+$ $2), \ldots, \mathfrak{R}-(\wp+\mathfrak{I})\}$.

In Lemma 1 , the sum $\delta(x)+\delta(y)$ is called as edge sum for each edge $x y \in E(\mathbb{G})$. This lemma will be used frequently in our derivations, as it keeps this sufficient to label the vertices of a network only to make the network S- $(a, 0)$ EAM total, if the edge sums are positive consecutive integers. The following result is also very pertinent as far as S- $(a, 0)$ EAM total networks are concerned.

Theorem 1. A simple network $G$ admits an $S$ - $(a, 0)-E A M$ total labelling $\Leftrightarrow G$ admits an $S-(a-|E(G)|+1,2)-E A M$ total labelling [36].

\subsection{Applications in Networking, Computer Science, and} Engineering. In software engineering, network labelling keeps on attaining an improved role in the security codes' encryption in order to encounter the attacks of trojans onto the precious data designed by hackers and also in designing of algorithm that helps the transmission of data to various networks and similar devices. The configurations of software in the encryption of their updated version is being improved by the use of reference labels and test ready labels nowadays. For connected components of networks in binary graphics, the mechanism which is predominantly nurturing the creation of clearer graphics involves labelling [37]. The study of magic labelling has been appearing to be more useful gradually in the data mining. The task of collection of data for the derivation of latest information gets more uncomplicated by designating equal weightage data as a single element. Resultantly, in organizations, the data mining task is becoming facile and more simplistic with far less consumption of time and effort due to the usage of magic labelling.

1.3.1. Networking. The primary hallmarks in networking are the functioning and optimization of the networks that demand management, construction, and concrete planning of networks at its base. Wireless and wired networking are two fundamental types of networking. The importance and large-scale usage of wired networking cannot be denied in the present era as well. The application of robust tools like network labelling is getting attention due to an escalation in the usage of wireless networking, in order to attain more precision in this field (see [38]). The modern era is of network communication whose part and parcel is radio transmission. The interference, making the job of channel assignment more complicated, is one of the major concerns in radio transmission. The transmission of concurrent networks that are constraint-free, admitting same instance surfacing, is the central reason of this unwanted interruption $[39,40]$. The magic labelling assists in the allotment of constant weights to the networks that are concurrent. Such interferences are eliminated by using this procedure. The radio labelling on networks is playing a tremendous part in the reduction of interference issue in wireless networking from the last decade or so. For the automatic routing in networks, the $(a, 0)$-antimagic labelling is particularly very useful. In this regard, a suitable constant edge-weight function is designed on a particular network, which helps routing for automatic detection of the succeeding node in the network (see [41]).

1.3.2. Telecommunication. In modern era, telecommunication involves most successful application of network labelling commercially [42]. In network telecommunication, a utility coverage region is split into a polygonal area described as a cell. Such a cell serves as a separate station. Using its radio transceiver, the base cell is designed to be a hub with the capacity to interface with other mobile stations. The defiance task here for the base cell is to facilitate with the ability to re-use utmost channels, avoiding any violation of the constraints. This challenge is being tackled by assigning a label to each user, while the communication loop of this user acquires a distinct label. Resultantly, any pair of communication terminals identifies the link label of connection path automatically by simple use of graceful antimagic or magic labelling. The label of the path specifies uniquely the two users which it interlinks conversely (see [43]).

1.3.3. Urban Planning. Consider the wheel $W_{6}$, the helm $H_{6}$, and prism $D_{5}$ in Figure 1 as a specific example. The edges of the networks $W_{6}$ and $H_{6}$ are labelled with consecutive labels ranging from 1 up to the size of the network such that the label appearing on all the vertices is distinct, i.e., we are provided here with the vertex antimagic labelling of the networks, whereas with edge weight 29 (constant), edge-magic labelling on $D_{5}$ is given $[44,45]$. As an example, the chambers are identified by point (vertices) and admissible pathways to approach these chambers are identified by edges, in a surveillance design of highly secured building. A total disturbance in the labelling will occur if a person attempts to breach a single legal pathway. The magic constant, in the scenario of design like $D_{5}$, gets disordered promptly in case of violation in the pathway. This disorder, through programming software, will abruptly alert to the security concerned that the legitimate pathway has been breached. Once such magic or antimagic labellings are designed on a network, they can be used for surveillance of all the networks having the same hubs and connections. Antimagic and magic labelling both are equally valuable in this regard. In urban planning, this is one of the large-scale 


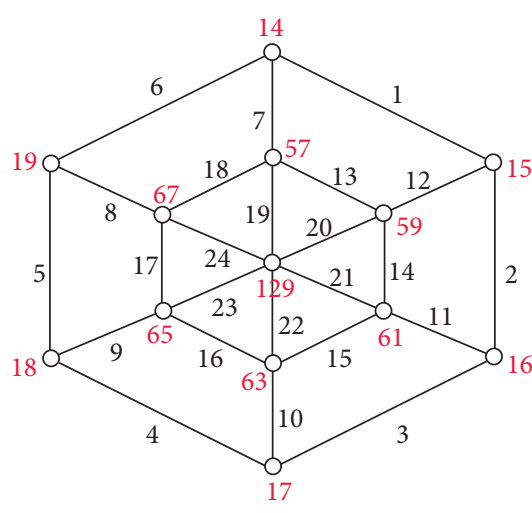

(a)

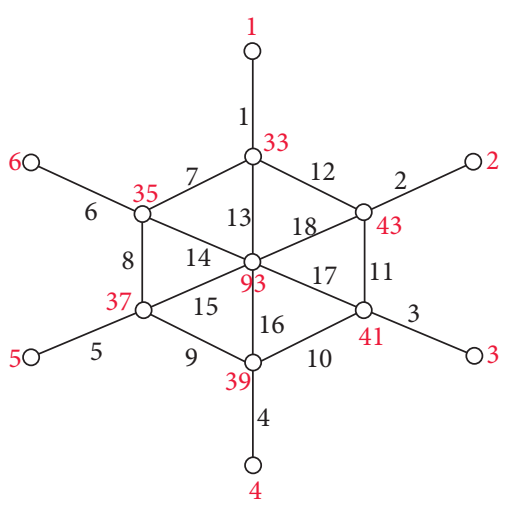

(b)

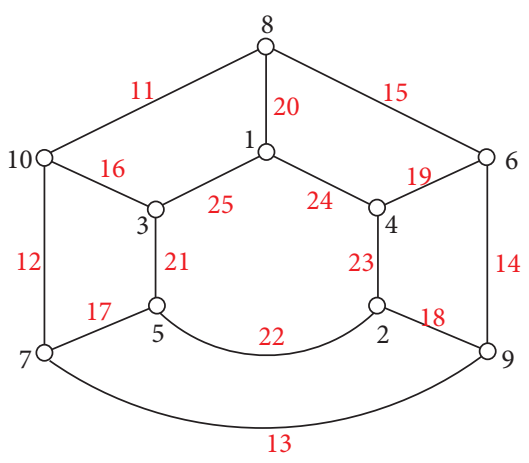

(c)

Figure 1: Vertex-antimagic total labelling of the wheel $W_{6}$ and helm $H_{6}$ and edge-magic labelling total of the prism $D_{5}$ [44,45].

usages of the concept of labelling. That is, as a model for surveillance of the extensively secure areas, these labellings perform their distinct role [46].

1.3.4. Robotics. The routing and functioning of inducted robots at places like restaurants and factories in the form of production lines and machine units derive assistance by making use of any such suitable labelling function. In order to keep robotic components kinetic or make them stationary, these labelling functions assist to opt which operation to be skipped at which instant and vice versa. The antimagic labelling and distance-based dimensions alike tools help to minimize the time and maximize the accuracy of robots in their routing [47]. In the industry, these tools are causing a massive reduction in the cost.

\section{Main Results}

This section contains our main findings. It is divided into four subsections further. In Section 2.1, the S- $(a, 0)$-EAM total labelling on the union of $K_{2, m}{ }^{\circ} C_{n}$ with copies of paths, the star, and the path shall be designed, whereas in Section 2.2 , we derive an S- $(a, 0)$-EAM total labelling on the rooted product of network $C_{n}$ and pancyclic networks $H_{1}$ and $H_{2}$ (planar also). Further, S- $(a, 0)$-EAM total labelling of planar pancyclic network $\Gamma_{n}$ and symmetric lattice networks $\mathbb{L}_{n}^{1}, \mathbb{L}_{n}^{2}$, and $\mathbb{L}_{n}^{3}$ shall be exhibited in Sections 2.3 and 2.4, respectively.

2.1. S- $(a, 0)$-EAM Total Labelling of the Disjoint Union of $C_{n}{ }^{\circ} K_{2, m}$ and $P_{n}, S_{n}$, and $m P_{2}$. Our main motivation to explore the findings in this section is the following open problem of Ngurah et al. [48].

Open Problem. For $n \geq 2$ and $m \geq 3$, is there any S- $(a, 0)$ EAM total labelling of $n K_{2, m}$ ?

In fact, the rooted product $C_{n}{ }^{\circ} \mathrm{K}_{2, \mathrm{~m}}$ contains $n$ copies of the complete bipartite network $K_{2, m}$. A cycle $C_{n}$ is a 2regular network of order $n$, whereas the complete bipartite network $K_{2, m}$ is class-wise regular in which one partitioned class of vertices is 2-regular and other is $m$-regular, where $\left|V\left(K_{2, m}\right)\right|=m+2$.

Theorem 2. For $2 \leq m \equiv 0(\bmod 2)$ and $3 \leq n \equiv 1(\bmod 2)$, the network $\left(C_{n} \circ K_{2, m}\right) \cup K_{1, n} \cup(n-1 / 2) K_{1}$ admits an $S-(a, 0)-E A M$ total labelling having $a=3 m n+8 n+2$.

Proof. Consider the network $G_{1} \cong\left(C_{n} \circ K_{2, m}\right) \cup K_{1, n} \cup(n-$ $1 / 2) K_{1}$ with connection scheme as below:

$$
\begin{aligned}
V\left(G_{1}\right) & =\left\{x_{j}^{i}: 1 \leq i \leq n 1 \leq j \leq m\right\} \cup\left\{y_{i}, z_{i}: 1 \leq i \leq n\right\} \\
& \cup\left\{p_{i}: 1 \leq i \leq n\right\} \cup\left\{c_{i}: 1 \leq i \leq(n-1 / 2)\right\} \cup\{c\}, \\
E\left(G_{1}\right) & =\left\{y_{i} x_{j}^{i}: 1 \leq i \leq n, 1 \leq j \leq m\right\} \\
& \cup\left\{z_{i} x_{j}^{i}: 1 \leq i \leq n, 1 \leq j \leq m\right\} \cup\left\{c p_{i}: 1 \leq i \leq n\right\} \\
& \cup\left\{y_{i} y_{i+1}: 1 \leq i \leq n-1\right\} \cup\left\{y_{1} y_{n}\right\} .
\end{aligned}
$$


Here, $\left|V\left(G_{1}\right)\right|=(7 n+2 m n+1 / 2)$ and $\left|E\left(G_{1}\right)\right|=2 n(m+$ 1). Define a labelling $f_{1}: V\left(G_{1}\right) \longrightarrow\{1,2, \ldots,(7 n+2 m n+$

(i) For $m=2$ :

$1 / 2)\}$ as

$$
f_{1}\left(x_{j}^{i}\right)= \begin{cases}\frac{5 n+i}{2}, & j=1, i \equiv 1(\bmod 2), 1 \leq i \leq n, \\ \frac{4 n+i}{2}, & j=1, i \equiv 0(\bmod 2), 2 \leq i \leq n-1, \\ n(m+2)-(i-1), & j=2,1 \leq i \leq n .\end{cases}
$$

(ii) For $m>2$ :

$$
f_{1}\left(x_{j}^{i}\right)= \begin{cases}\frac{5 n+i}{2}, & j=\frac{m}{2}, i \equiv 1(\bmod 2), 1 \leq i \leq n, \\ \frac{4 n+i}{2}, & j=\frac{m}{2}, i \equiv 0(\bmod 2), 2 \leq i \leq n-1, \\ n(m+2)-(i-1), & j=\frac{m}{2}+1,1 \leq i \leq n, \\ n(j+3)-(i-1), & 1 \leq i \leq n, 1 \leq j \leq \frac{m}{2}-1 . \\ n j-(i-1)+n: & 1 \leq i \leq n, \frac{m}{2}+2 \leq j \leq m .\end{cases}
$$

(iii) The remaining labels for $m \geq 2$ are as follows:

$$
\begin{aligned}
& f_{1}\left(y_{i}\right)= \begin{cases}\frac{i+2 n+1}{2}, & i \equiv 1(\bmod 2), 1 \leq i \leq n, \\
\frac{i+3 n+1}{2}, & i \equiv 0(\bmod 2), 2 \leq i \leq n-1,\end{cases} \\
& f_{1}\left(z_{i}\right)= \begin{cases}n(m+1)+\frac{i+2 n+1}{2}, & i \equiv 1(\bmod 2), 1 \leq i \leq n, \\
n(m+1)+\frac{i+3 n+1}{2}, & i \equiv 0(\bmod 2), 2 \leq i \leq n-1 .\end{cases} \\
& f_{1}\left(p_{i}\right)=i, \quad 1 \leq i \leq n, \quad \\
& f_{1}(c)=\frac{7 n+2 m n+1}{2}, \quad \\
& f_{1}\left(c_{i}\right)=m n+3 n+i, \quad 1 \leq i \leq \frac{n-1}{2} .
\end{aligned}
$$


All edge sums generated by the labelling scheme $f_{1}$ constitute a sequence of consecutive integer $(5 n+3 / 2),(5 n+$ $5 / 2),(5 n+7 / 2), \ldots,(4 m n+9 n+1 / 2)$. So, by Lemma $1, f_{1}$ extends to an S- $(a, 0)$-EAM total labelling of the network $G_{1}$ having magic constant $a=3 m n+8 n+2$.
Theorem 3. For $3 \leq m \equiv 1(\bmod 2)$ and $3 \leq n \equiv 1(\bmod 2)$, the network $\left(C_{n} \circ K_{2, m}\right) \cup K_{1, n} \cup(n-1 / 2) K_{1}$ admits an $S-(a, 0)-E A M$ total labelling with $a=3 m n+8 n+2$.

Proof. Consider $G_{2} \cong\left(C_{n} \circ K_{2, m}\right) \cup K_{1, n} \cup(n-1 / 2) K_{1}$, a network with odd $m$ as follows:

$$
\begin{aligned}
V\left(G_{2}\right)= & \left\{x_{j}^{i}: 1 \leq i \leq n, 1 \leq j \leq m\right\} \cup\left\{y_{i}, z_{i}: 1 \leq i \leq n\right\} \\
& \cup\left\{p_{i}: 1 \leq i \leq n\right\} \cup\left\{c_{i}: 1 \leq i \leq(n-1 / 2)\right\} \cup\{c\}, \\
E\left(G_{2}\right)= & \left\{y_{i} x_{j}^{i}: 1 \leq i \leq n, 1 \leq j \leq m\right\} \cup\left\{z_{i} x_{j}^{i}: 1 \leq i \leq n, 1 \leq j \leq m\right\} \cup\left\{c p_{i}: 1 \leq i \leq n\right\} \\
& \cup\left\{y_{i} y_{i+1}: 1 \leq i \leq n-1\right\} \cup\left\{y_{1} y_{n}\right\} .
\end{aligned}
$$

Here, $\quad\left|V\left(G_{2}\right)\right|=(7 n+2 m n+1 / 2) \quad$ and $\left|E\left(G_{2}\right)\right|=2 n(m+1)$. We define a labelling $f_{2}: V\left(G_{2}\right) \longrightarrow\{1,2, \ldots,(7 n+2 m n+1 / 2)\}$ as follows:

$$
f_{2}\left(x_{j}^{i}\right)= \begin{cases}\frac{5 n+i}{2}, & j=2, i \equiv 1(\bmod 2), 1 \leq i \leq n, \\ \frac{4 n+i}{2}, & j=2, i \equiv 0(\bmod 2), 2 \leq i \leq n-1, \\ n(m+2)-(i-1), & j=3,1 \leq i \leq n, \\ 4 n-(i-1), & j=1,1 \leq i \leq n .\end{cases}
$$

(ii) For $m>3$ :

$$
f_{2}\left(x_{j}^{i}\right)= \begin{cases}\frac{5 n+i}{2}, & j=\frac{m+1}{2}, i \equiv 1(\bmod 2), 1 \leq i \leq n, \\ \frac{4 n+i}{2}, & j=\frac{m+1}{2}, i \equiv 0(\bmod 2), 2 \leq i \leq n-1, \\ n(m+2)-(i-1), & j=\frac{m+3}{2}, 1 \leq i \leq n, \\ n(j+3)-(i-1), & 1 \leq i \leq n, 1 \leq j \leq \frac{m-1}{2} . \\ n j-(i-1)+n: & 1 \leq i \leq n, \frac{m+5}{2} \leq j \leq m .\end{cases}
$$


(iii) The remaining labels for $m \geq 3$ are as follows:

$$
\begin{aligned}
& f_{2}\left(y_{i}\right)= \begin{cases}\frac{i+2 n+1}{2}, & i \equiv 1(\bmod 2), 1 \leq i \leq n, \\
\frac{i+3 n+1}{2}, & i \equiv 0(\bmod 2), 2 \leq i \leq n-1,\end{cases} \\
& f_{2}\left(z_{i}\right)= \begin{cases}n(m+1)+\frac{i+2 n+1}{2}, & i \equiv 1(\bmod 2), 1 \leq i \leq n, \\
n(m+1)+\frac{i+3 n+1}{2}, & i \equiv 0(\bmod 2), 2 \leq i \leq n-1,\end{cases} \\
& f_{2}\left(p_{i}\right)=i, \quad 1 \leq i \leq n, \\
& f_{2}(c)=\frac{7 n+2 m n+1}{2}, \\
& f_{2}\left(c_{i}\right)=m n+3 n+i, \quad 1 \leq i \leq \frac{n-1}{2} .
\end{aligned}
$$

All edge sums generated by the above labelling scheme $f_{2}$ constitute a sequence of consecutive integer $(5 n+3 / 2),(5 n+5 / 2),(5 n+7 / 2), \ldots,(4 m n+9 n+1 / 2)$. Ву Lemma $1, f_{2}$ extends to an S- $(a, 0)$-EAM total labelling of the network $G_{2}$ having $a=3 m n+8 n+2$.

Theorem 4. For $2 \leq m \equiv 0(\bmod 2)$ and $3 \leq n \equiv 1(\bmod 2)$, the network $\left(C_{n} \circ K_{2, m}\right) \cup n P_{2}$ admits an $S-(a, 0)-E A M$ total labelling with $a=(6 m n+17 n+3 / 2)$.

Proof. Let $G_{3} \cong\left(C_{n} \circ K_{2, m}\right) \cup n P_{2}$ be a network for $n n \equiv 1(\bmod 2)$ with vertices:

$$
f_{3}\left(x_{j}^{i}\right)= \begin{cases}\frac{1}{2}(5 n+i), & j=1,1 \leq i \leq n, i \equiv 1(\bmod 2), \\ \frac{1}{2}(4 n+i), & j=1, i \equiv 0(\bmod 2), 2 \leq i \leq n-1, \\ n(m+2)-(i-1), & j=2,1 \leq i \leq n .\end{cases}
$$

(ii) For $m>2$ :

$$
\begin{aligned}
V\left(G_{3}\right) & =\left\{x_{j}^{i}: 1 \leq i \leq n, 1 \leq j \leq m\right\} \cup\left\{y_{i}, z_{i}: 1 \leq i \leq n\right\} \\
& \cup\left\{p_{i}, q_{i}: 1 \leq i \leq n\right\}, \\
E\left(G_{3}\right) & =\left\{y_{i} x_{j}^{i}: 1 \leq i \leq n, 1 \leq j \leq m\right\} \cup\left\{z_{i} x_{j}^{i}: 1 \leq i \leq n, 1 \leq j \leq m\right\} \\
& \cup\left\{p_{i} q_{i}: 1 \leq i \leq n\right\} \\
& \cup\left\{y_{i} y_{i+1}: 1 \leq i \leq n-1\right\} \cup\left\{y_{1} y_{n}\right\} .
\end{aligned}
$$

Here, $\left|V\left(G_{3}\right)\right|=n(m+4)$ and $\left|E\left(G_{3}\right)\right|=2 n(m+1)$. A labelling function $f_{3}: V\left(G_{3}\right) \longrightarrow\{1,2, \ldots, n(m+4)\}$ is defined as follows:

(i) For $m=2$ :

$$
f_{3}\left(x_{j}^{i}\right)= \begin{cases}\frac{1}{2}(5 n+i), & j=\frac{m}{2}, i \equiv 1(\bmod 2), 1 \leq i \leq n, \\ \frac{1}{2}(4 n+i), & j=\frac{m}{2}, i \equiv 0(\bmod 2), 2 \leq i \leq n-1, \\ n(m+2)-(i-1), & j=\frac{m}{2}+1,1 \leq i \leq n, \\ n(j+3)-(i-1), & 1 \leq i \leq n, 1 \leq j \leq \frac{m}{2}-1 . \\ n j-(i-1)+n: & 1 \leq i \leq n, \frac{m}{2}+2 \leq j \leq m .\end{cases}
$$


(iii) The remaining labels for $m \geq 2$ are as follows:

$$
\begin{aligned}
& f_{3}\left(y_{i}\right)= \begin{cases}\frac{i+2 n+1}{2}, & i \equiv 1(\bmod 2), 1 \leq i \leq n, \\
\frac{i+3 n+1}{2}, & i \equiv 0(\bmod 2), 2 \leq i \leq n-1,\end{cases} \\
& f_{3}\left(z_{i}\right)= \begin{cases}n(m+1)+\frac{i+2 n+1}{2}, & i \equiv 1(\bmod 2), 1 \leq i \leq n, \\
n(m+1)+\frac{i+3 n+1}{2}, & i \equiv 0(\bmod 2), 2 \leq i \leq n-1 .\end{cases} \\
& f_{3}\left(q_{i}\right)= \begin{cases}n(m+1)+\frac{5 n-i+2}{2}: & i \equiv 1(\bmod 2), 1 \leq i \leq n, \\
n(m+1)+\frac{6 n-i+2}{2}: & i \equiv 0(\bmod 2), 2 \leq i \leq n-1,\end{cases} \\
& f_{3}\left(p_{i}\right)=i, \quad 1 \leq i \leq n .
\end{aligned}
$$

All edge sums generated by the above labelling scheme $f_{3}$ constitute a sequence of consecutive integer $(5 n+3 / 2),(5 n+$ $5 / 2),(5 n+7 / 2), \ldots,(4 m n+9 n+1 / 2)$. So, by Lemma $1, f_{3}$ extends to an S- $(a, 0)$-EAM total labelling of the network $G_{3}$ admitting magic constant $a=(6 m n+17 n+3 / 2)$.

Theorem 5. For $3 \leq m \equiv 1(\bmod 2)$ and $3 \leq n \equiv 1(\bmod 2)$, the network $\left(C_{n} \circ K_{2, m}\right) \cup n P_{2}$ admits an $S-(a, 0)-E A M$ total labelling with $a=(6 m n+17 n+3 / 2)$.

Proof. Consider the network $G_{4} \cong\left(C_{n} \circ K_{2, m}\right) \cup n P_{2}$, for odd $m$ with the construction:

$$
\begin{aligned}
V\left(G_{4}\right) & =\left\{x_{j}^{i}: 1 \leq i \leq n, 1 \leq j \leq m\right\} \cup\left\{y_{i}, z_{i}: 1 \leq i \leq n\right\} \\
& \cup\left\{p_{i}, q_{i}: 1 \leq i \leq n\right\}, \\
E\left(G_{4}\right) & =\left\{y_{i} x_{j}^{i}: 1 \leq i \leq n, 1 \leq j \leq m\right\} \\
& \cup\left\{z_{i} x_{j}^{i}: 1 \leq i \leq n, 1 \leq j \leq m\right\} \cup\left\{p_{i} q_{i}: 1 \leq i \leq n\right\} \\
& \cup\left\{y_{i} y_{i+1}: 1 \leq i \leq n-1\right\} \cup\left\{y_{1} y_{n}\right\} .
\end{aligned}
$$

Here, $\left|V\left(G_{4}\right)\right|=n(m+4)$ and $\left|E\left(G_{4}\right)\right|=2 n(m+1)$. Define a function $f_{4}: V\left(G_{4}\right) \longrightarrow\{1,2, \ldots, n(m+4)\}$ as follows:

(i) For $m=3$ :

(ii) For $m>3$ : 
(iii) The remaining labels for $m \geq 3$ are as follows:

$$
\begin{aligned}
& f_{4}\left(y_{i}\right)= \begin{cases}\frac{i+2 n+1}{2}, & i \equiv 1(\bmod 2), 1 \leq i \leq n, \\
\frac{i+3 n+1}{2}, & i \equiv 0(\bmod 2), 2 \leq i \leq n-1,\end{cases} \\
& f_{4}\left(z_{i}\right)= \begin{cases}n(m+1)+\frac{i+2 n+1}{2}, & i \equiv 1(\bmod 2), 1 \leq i \leq n, \\
n(m+1)+\frac{i+3 n+1}{2}, & i \equiv 0(\bmod 2), 2 \leq i \leq n-1,\end{cases} \\
& f_{4}\left(q_{i}\right)= \begin{cases}n(m+1)+\frac{5 n-i+2}{2}, & i \equiv 1(\bmod 2), 1 \leq i \leq n, \\
n(m+1)+\frac{6 n-i+2}{2}, & i \equiv 0(\bmod 2), 2 \leq i \leq n-1,\end{cases} \\
& f_{4}\left(p_{i}\right)=i, \quad 1 \leq i \leq n .
\end{aligned}
$$

All edge sums generated by the above labelling scheme $f_{4}$ constitute a sequence of consecutive integer $(5 n+3 / 2),(5 n+5 / 2),(5 n+7 / 2), \ldots,(4 m n+9 n+1 / 2)$. So, by Lemma $1, f_{4}$ extends to an S- $(a, 0)$-EAM total labelling of the network $G_{4}$ admitting $a=(6 m n+17 n+3 / 2)$.

Theorem 6. For $2 \leq m \equiv 0(\bmod 2)$ and $3 \leq n \equiv 1(\bmod 2)$, the network $\left(C_{n} \circ K_{2, m}\right) \cup P_{n+1}$ admits an $S$ - $(a, 0)-E A M$ total labelling with $a=(6 m n+17 n+3 / 2)$.

Proof. Consider the network $G_{5} \cong\left(C_{n} \circ K_{2, m}\right) \cup P_{n+1}$, for $n \geq 3$ odd, with connections:

$$
\begin{aligned}
V\left(G_{5}\right) & =\left\{x_{j}^{i}: 1 \leq i \leq n, 1 \leq j \leq m\right\} \\
& \cup\left\{y_{i}, z_{i}: 1 \leq i \leq n\right\} \cup\left\{p_{i}: 1 \leq i \leq n+1\right\}, \\
E\left(G_{5}\right)= & \left\{y_{i} x_{j}^{i}: 1 \leq i \leq n, 1 \leq j \leq m\right\} \\
& \cup\left\{z_{i} x_{j}^{i}: 1 \leq i \leq n, 1 \leq j \leq m\right\} \cup\left\{y_{1} y_{n}\right\} \\
& \cup\left\{p_{i} p_{i+1}: 1 \leq i \leq n\right\} \cup\left\{y_{i} y_{i+1}: 1 \leq i \leq n-1\right\} .
\end{aligned}
$$

Here, $\left|V\left(G_{5}\right)\right|=3 n+m n+1$ and $\left|E\left(G_{5}\right)\right|=2 n(m+1)$. A labelling $f_{5}: V\left(G_{5}\right) \longrightarrow\{1,2, \ldots, m n+3 n+1\}$ is defined as follows:

(i) For $m=2$ :

(ii) For $m>2$ :

$$
f_{5}\left(x_{j}^{i}\right)= \begin{cases}\frac{1}{2}(4 n+i+1), & j=1, i \equiv 1(\bmod 2), 1 \leq i \leq n, \\ \frac{1}{2}(3 n+i+1), & j=1, i \equiv 0(\bmod 2), 2 \leq i \leq n-1, \\ \frac{1}{2}(2 m n+3 n-2 i+3), & j=2,1 \leq i \leq n .\end{cases}
$$

$$
f_{5}\left(x_{j}^{i}\right)= \begin{cases}\frac{1}{2}(4 n+i+1), & j=\frac{m}{2}, i \equiv 1(\bmod 2), 1 \leq i \leq n, \\ \frac{1}{2}(3 n+i+1), & j=\frac{m}{2}, i \equiv 0(\bmod 2), 2 \leq i \leq n-1, \\ \frac{1}{2}(2 m n+3 n-2 i+3), & j=\frac{m}{2}+1,1 \leq i \leq n, \\ \frac{1}{2}(2 n j+5 n-2 i+3), & 1 \leq i \leq n, 1 \leq j \leq \frac{m}{2}-1, \\ \frac{1}{2}(2 n j+n-2 i+3): & 1 \leq i \leq n, \frac{m}{2}+2 \leq j \leq m .\end{cases}
$$


(iii) The remaining labels for $m \geq 2$ are as follows:

$$
\begin{aligned}
& f_{5}\left(y_{i}\right)= \begin{cases}\frac{n+i+2}{2}, & i \equiv 1(\bmod 2), 1 \leq i \leq n, \\
\frac{2 n+i+2}{2}, & i \equiv 0(\bmod 2), 2 \leq i \leq n-1,\end{cases} \\
& f_{5}\left(z_{i}\right)= \begin{cases}n(m+1)+\frac{n+i+2}{2}, & i \equiv 1(\bmod 2), 1 \leq i \leq n, \\
n(m+1)+\frac{2 n+i+2}{2}, & i \equiv 0(\bmod 2), 2 \leq i \leq n-1,\end{cases} \\
& f_{5}\left(p_{i}\right)= \begin{cases}\frac{i+1}{2}, & i \equiv 1(\bmod 2), 1 \leq i \leq n, \\
\frac{1}{2}(2 m n+5 n+i+1), & i \equiv 0(\bmod 2), 2 \leq i \leq n+1 .\end{cases}
\end{aligned}
$$

All edge sums generated by the above labelling scheme $f_{5}$ constitute a sequence of consecutive integer $(3 n+5 / 2)$, $(3 n+7 / 2),(3 n+9 / 2), \ldots,(4 m n+7 n+3 / 2)$. Therefore, by Lemma $1, f_{5}$ extends to an S- $(a, 0)$-EAM total labelling of the network $G_{5}$ admitting magic constant $a=(6 m n+13 n+$ $7 / 2$ ).

Theorem 7. For $3 \leq m \equiv 1(\bmod 2)$ and $3 \leq n \equiv 1(\bmod 2)$, the network $\left(C_{n} \circ K_{2, m}\right) \cup P_{n+1}$ admits an $S-(a, 0)-E A M$ total labelling with $a=(6 m n+13 n+7 / 2)$.

Proof. Consider the network $G_{6} \cong\left(C_{n} \circ K_{2, m}\right) \cup P_{n+1}$, for both $m$ and $n \geq 3$, as follows:

$$
\begin{aligned}
V\left(G_{6}\right) & =\left\{x_{j}^{i}: 1 \leq i \leq n, 1 \leq j \leq m\right\} \\
& \cup\left\{y_{i}, z_{i}: 1 \leq i \leq n\right\} \cup\left\{p_{i}: 1 \leq i \leq n+1\right\}, \\
E\left(G_{6}\right) & =\left\{y_{i} x_{j}^{i}: 1 \leq i \leq n, 1 \leq j \leq m\right\} \\
& \cup\left\{z_{i} x_{j}^{i}: 1 \leq i \leq n, 1 \leq j \leq m\right\} \cup\left\{y_{1} y_{n}\right\} \\
& \cup\left\{p_{i} p_{i+1}: 1 \leq i \leq n\right\} \cup\left\{y_{i} y_{i+1}: 1 \leq i \leq n-1\right\} .
\end{aligned}
$$

Here, $\left|V\left(G_{6}\right)\right|=3 n+m n+1$ and $\left|E\left(G_{6}\right)\right|=2 n(m+1)$. A labelling $f_{6}: V\left(G_{6}\right) \longrightarrow\{1,2, \ldots, 3 n+m n+1\}$ is designed as follows:

(i) For $m=3$ :

$$
f_{6}\left(x_{j}^{i}\right)= \begin{cases}\frac{1}{2}(4 n+i+1), & j=2, i \equiv 1(\bmod 2), 1 \leq i \leq n, \\ \frac{1}{2}(3 n+i+1), & j=2, i \equiv 0(\bmod 2), 2 \leq i \leq n-1, \\ \frac{1}{2}(2 m n+3 n-2 i+3), & j=3,1 \leq i \leq n, \\ \frac{1}{2}(7 n-2 i+3), & j=1,1 \leq i \leq n .\end{cases}
$$

(ii) For $m>3$ :

$$
f_{6}\left(x_{j}^{i}\right)= \begin{cases}\frac{1}{2}(4 n+i+1), & j=\frac{m+1}{2}, i \equiv 1(\bmod 2), 1 \leq i \leq n, \\ \frac{1}{2}(3 n+i+1), & j=\frac{m+1}{2}, i \equiv 0(\bmod 2), 2 \leq i \leq n-1, \\ \frac{1}{2}(2 m n+3 n-2 i+3), & j=\frac{m+3}{2}, 1 \leq i \leq n, \\ \frac{1}{2}(2 n j+5 n-2 i+3), & 1 \leq i \leq n, 1 \leq j \leq \frac{m-1}{2}, \\ \frac{1}{2}(2 n j+n-2 i+3), & 1 \leq i \leq n, \frac{m+5}{2} \leq j \leq m .\end{cases}
$$


(iii) The remaining labels for $m \geq 3$ are as follows:

$$
\begin{aligned}
& f_{6}\left(y_{i}\right)= \begin{cases}\frac{n+i+2}{2}, & i \equiv 1(\bmod 2), 1 \leq i \leq n, \\
\frac{2 n+i+2}{2}, & i \equiv 0(\bmod 2), 2 \leq i \leq n-1,\end{cases} \\
& f_{6}\left(z_{i}\right)= \begin{cases}n(m+1)+\frac{n+i+2}{2}, & i \equiv 1(\bmod 2), 1 \leq i \leq n, \\
n(m+1)+\frac{2 n+i+2}{2}, & i \equiv 0(\bmod 2), 2 \leq i \leq n-1,\end{cases} \\
& f_{6}\left(p_{i}\right)= \begin{cases}\frac{i+1}{2}, & i \equiv 1(\bmod 2), 1 \leq i \leq n, \\
\frac{1}{2}(2 m n+5 n+i+1), & i \equiv 0(\bmod 2), 2 \leq i \leq n+1 .\end{cases}
\end{aligned}
$$

The edge sums generated by the above labelling scheme $f_{6}$ constitute a sequence of consecutive integer $(3 n+5 / 2)$, $(3 n+7 / 2),(3 n+9 / 2), \ldots,(4 m n+7 n+3 / 2)$. By Lemma 1 , $f_{6}$ extends to an S- $(a, 0)$-EAM total labelling of the network $G_{6}$ having magic constant $a=(6 m n+13 n+7 / 2)$.

Observations. The network $C_{n}$ is S- $(a, 0)$-EAM total for $n \equiv 1(\bmod 2)$ only [8], pointing out that $C_{4}$ is not $S-(a, 0)$ EAM total. The networks in Theorems 2, 4, and 6 contain interesting substructures. Keeping $m=2$ fixed for these results, S- $(a, 0)$-EAM total families of networks involving $n$ copies of $C_{4}$ can be obtained. For instance, S- $(a, 0)$-EAM total labelling (again by using Lemma 1) of $\left(C_{5} \circ C_{4}\right) \cup K_{1,5} \cup 2 K_{1},\left(C_{5} \circ C_{4}\right) \cup 5 P_{2}$, and $\left(C_{5} \circ C_{4}\right) \cup P_{6}$ is presented in Figure 2.

The following results from Theorems 2-7 are direct consequences of Theorem 1.

Theorem 8. $\forall m \geq 2$ and $3 \leq n \equiv 1(\bmod 2),\left(C_{n} \circ K_{2, m}\right) \cup$ $K_{1, n} \cup(n-1 / 2) K_{1}$ admits an $S-(m n+6 n+3,2)-E A M$ total labelling.

Theorem 9. $\forall m \geq 2$ and $3 \leq n \equiv 1(\bmod 2), \quad\left(C_{n} \circ K_{2, m}\right) \cup$ $n P_{2}$ admits an $S-((2 m n+13 n+5 / 2), 2)-E A M$ total labelling.

Theorem 10. $\forall m \geq 2$ and $3 \leq n \equiv 1(\bmod 2),\left(C_{n} \circ K_{2, m}\right) \cup$ $P_{n+1}$ admits an $S-((2 m n+9 n+9 / 2), 2)-E A M$ total labelling.

2.2. S-(a, 0)-EAM Total Labelling of Rooted Product of Pancyclic Networks with $C_{n}$. The present section deals with
S- $(a, 0)$-EAM total labelling of the rooted product of two specific planar non-isomorphic pancyclic networks and the cycle $C_{n}$.

A specific pancyclic network $H_{1}$ is defined as follows.

Definition 5. $H_{1}$ is a pancyclic network having the following construction.

$$
\begin{aligned}
V\left(H_{1}\right)= & \left\{y, x_{1}, x_{2}, x_{3}, x_{4}, x_{5}, x_{6}, z\right\}, \\
E\left(H_{1}\right)= & \left\{x_{1} x_{3}, x_{3} x_{5}, x_{2} x_{4}, x_{4} x_{6}, x_{1} x_{2},\right. \\
& \left.\cdot x_{3} x_{4}, x_{5} x_{6}, x_{2} x_{3}, x_{4} x_{5}\right\} \cup\left\{y x_{1}, y x_{2}, z x_{5}, z x_{6}\right\} .
\end{aligned}
$$

Theorem 11. For $n \equiv 1(\bmod 2)$, the rooted product $C_{n} \circ H_{1}$ admits an $S-(a, 0)-E A M$ total labelling having magic constant $a=(45 n+3 / 2)$.

Proof

(i) For $n=1, \quad C_{1} \circ H_{1} \cong H_{1}$. The vertex labelling $\left\{y, x_{1}, x_{2}, x_{3}, x_{4}, x_{5}, x_{6}, y, z: 2,1,4,3,6,5,8,7\right\}$ extends to an S- $(24,0)$-EAM total labelling of $C_{n} \circ H_{1}$, by Lemma 1 .

(ii) For $n \geq 3$.

Consider the network $C_{n} \circ H_{1}$ with $\left|V\left(C_{n} \circ H_{1}\right)\right|=8 n$ and $\left|E\left(C_{n} \circ H_{1}\right)\right|=14 n$ connected as per the following scheme:

$$
\begin{aligned}
V\left(C_{n} \circ H_{1}\right)= & \left\{x_{i}, v_{i}: 1 \leq i \leq n\right\} \cup\left\{y_{i}, z_{i}, w_{i}: 1 \leq i \leq 2 n\right\}, \\
E\left(C_{n} \circ H_{1}\right)= & \left\{y_{i} y_{i+1}, w_{i} w_{i+1}, z_{i} z_{i+1}: 1 \leq i \leq 2 n-1, i \equiv 1(\bmod 2)\right\} \\
& \cup\left\{y_{i} z_{i}, z_{i} w_{i}: 1 \leq i \leq 2 n\right\} \cup\left\{x_{i} x_{i+1}: 1 \leq i \leq n-1\right\} \\
& \cup\left\{x_{1} x_{n}\right\} \cup\left\{z_{i} y_{i+1}, w_{i} z_{i+1}: 1 \leq i \leq 2 n-1, i \equiv 1(\bmod 2)\right\} \\
& \cup\left\{x_{i} y_{2 i-1}, x_{i} y_{2 i}, v_{i} w_{2 i-1}, v_{i} w_{2 i}: 1 \leq i \leq n\right\} .
\end{aligned}
$$




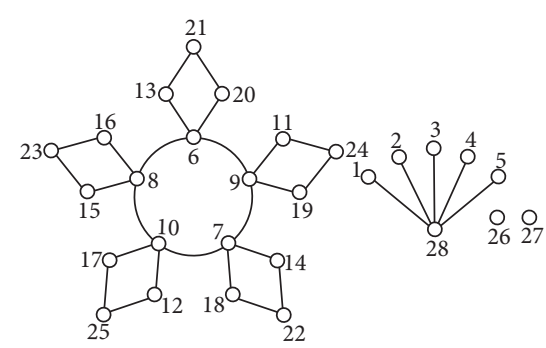

(a)

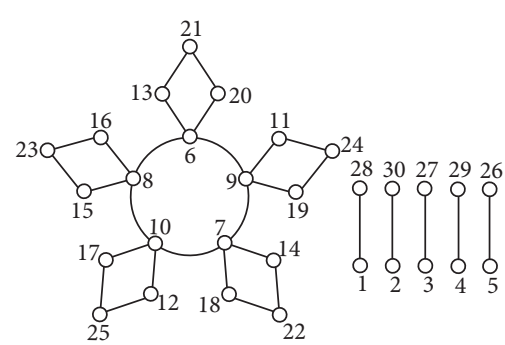

(b)

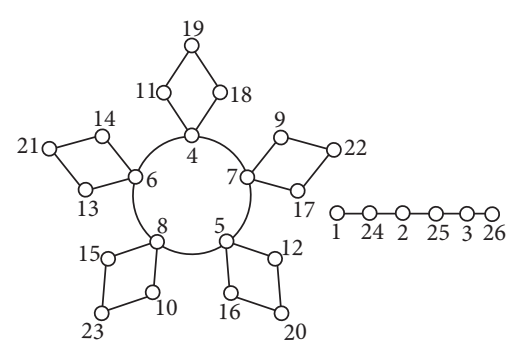

(c)

Figure 2: An S- $(a, 0)$-EAM total labelling (using Lemma 1) of the cyclic networks (a) $\left(C_{5} \circ C_{4}\right) \cup K_{1,5} \cup 2 K_{1}$, (b) $\left(C_{5} \circ C_{4}\right) \cup 5 P_{2}$, and (c) $\left(C_{5} \circ C_{4}\right) \cup P_{6}$.

Consider a labelling $\psi_{1}: V\left(C_{n} \circ H_{1}\right) \longrightarrow\{1,2, \ldots, \mid V$

$\left.\left(C_{n} \circ H_{1}\right) \mid=8 n\right\}$ defined as

$$
\begin{aligned}
& \psi_{1}\left(x_{i}\right)= \begin{cases}\frac{i+1}{2}, & i \equiv 1(\bmod 2), 1 \leq i \leq n, \\
\frac{i+n+1}{2}, & i \equiv 0(\bmod 2), 2 \leq i \leq n-1,\end{cases} \\
& \psi_{1}\left(y_{i}\right)= \begin{cases}\frac{6 n-i+2}{2}, & i \equiv 0(\bmod 2), 2 \leq i \leq 2 n, \\
\frac{6 n+i+1}{4}, & i \equiv 1(\bmod 4), 1 \leq i \leq 2 n-1, \\
\frac{4 n+i+1}{4}, & i \equiv 3(\bmod 4), 3 \leq i \leq 2 n-3,\end{cases} \\
& \psi_{1}\left(z_{i}\right)= \begin{cases}\frac{16 n+i}{4}, & i \equiv 0(\bmod 4), 4 \leq i \leq 2 n-2, \\
\frac{12 n+i+3}{4}, & i \equiv 1(\bmod 4), 1 \leq i \leq 2 n-1, \\
\frac{18 n+i}{4}, & i \equiv 2(\bmod 4), 2 \leq i \leq 2 n, \\
\frac{14 n+i+3}{4}, & i \equiv 3(\bmod 4), 3 \leq i \leq 2 n-3,\end{cases} \\
& \psi_{1}\left(w_{i}\right)= \begin{cases}\frac{12 n-i+1}{2}, & i \equiv 1(\bmod 2), 1 \leq i \leq 2 n-1, \\
\frac{26 n+i+2}{4}, & i \equiv 0(\bmod 4), 4 \leq i \leq 2 n-2, \\
\frac{24 n+i+2}{4}, & i \equiv 2(\bmod 4), 2 \leq i \leq 2 n,\end{cases} \\
& \psi_{1}\left(v_{i}\right)= \begin{cases}\frac{15 n+i}{2}, & i \equiv 1(\bmod 2), 1 \leq i \leq n, \\
\frac{14 n+i}{2}, & i \equiv 0(\bmod 2), 2 \leq i \leq n-1 .\end{cases}
\end{aligned}
$$


All edge sums generated by the above labelling scheme $\psi_{1}$ form a sequence of consecutive integer $(n+3 / 2),(n+5 / 2),(n+7 / 2), \ldots,(29 n+1 / 2)$. Therefore, by Lemma $1, \psi_{1}$ extends to an S- $(a, 0)$-EAM total labelling of the network $C_{n} \circ H_{1}$ having magic constant $(45 n+3 / 2)$.
Definition 6. We define a pancyclic network $H_{2} ¥ H_{1}$ having vertex set:

$$
\begin{aligned}
& V\left(H_{2}\right)=\left\{y, x_{1}, x_{2}, x_{3}, x_{4}, x_{5}, x_{6}, z\right\}, \\
& E\left(H_{2}\right)=\left\{x_{1} x_{3}, x_{3} x_{5}, x_{2} x_{4}, x_{4} x_{6}, x_{1} x_{2}, x_{5} x_{6}, x_{2} x_{3}, x_{4} x_{5}\right\} \cup\left\{y x_{1}, y x_{2}, z x_{5}, z x_{6}, y z\right\} .
\end{aligned}
$$

Theorem 12. For $n \equiv 1(\bmod 2)$, the rooted product $C_{n} \circ H_{2}$ admits an $S-(a, 0)$-EAM total labelling with magic constant $a=(45 n+3 / 2)$.

\section{Proof}

(i) For $n=1, C_{1} \circ H_{2} \cong H_{2}$.

(ii) For $n \geq 3$.

Consider $\mathrm{C}_{n} \circ \mathrm{H}_{2}$ with $\left|V\left(\mathrm{C}_{n} \circ \mathrm{H}_{2}\right)\right|=8 n$ and $\left|E\left(C_{n} \circ H_{2}\right)\right|=14 n$ with the following connection:

$$
V\left(C_{n} \circ H_{2}\right)=\left\{x_{i}, v_{i}: 1 \leq i \leq n\right\} \cup\left\{w_{i}, y_{i}, z_{i}: 1 \leq i \leq 2 n\right\},
$$$$
E\left(P_{n} \circ H_{2}\right)=\left\{y_{i} y_{i+1}, w_{i} w_{i+1}: 1 \leq i \leq 2 n-1, i \equiv 1(\bmod 2)\right\}
$$$$
\cup\left\{y_{i} z_{i}, z_{i} w_{i}: 1 \leq i \leq 2 n\right\} \cup\left\{x_{i} x_{i+1}: 1 \leq i \leq n-1\right\} \cup\left\{x_{1} x_{n}\right\}
$$$$
\begin{aligned}
& \cup\left\{z_{i} y_{i+1}, w_{i} z_{i+1}: 1 \leq i \leq 2 n-1, i \equiv 1(\bmod 2)\right\} \\
& \cup\left\{x_{i} y_{2 i-1}, x_{i} y_{2 i}, v_{i} w_{2 i-1}, v_{i} w_{2 i}, x_{i} v_{i}: 1 \leq i \leq n\right\} .
\end{aligned}
$$

The labelling scheme for $n=1$ and $n \geq 3$ is the same as $\psi_{1}$ designed in Theorem 11.

A direct derivation from Theorem 1 is given as follows.

Theorem 13. For $n \equiv 1(\bmod 2), C_{n} \circ H_{1}$ and $C_{n} \circ H_{2}$ are $S-((17 n+5 / 2), 2)-E A M$ total.

2.3. S- $(a, 0)-E A M$ Total Labelling of a Pancyclic Class of Networks: Extension of a Result Appearing in [29]. In [29], Baig et al. provided a result regarding S- $(a, 0)$-EAM total labelling of a pancyclic class of networks involving chains of cycle $C_{4}$. Here, we shall introduce a pancyclic family of networks involving chains of cycle $C_{6}$, while our point of convergence is the S- $(a, 0)$-EAM total labelling of this class. Thus, we further extend the results of Baig et al. [29].

Definition 7. The pancyclic network $\Gamma_{n}$ is a network with order $\left|V\left(\Gamma_{n}\right)\right|=6 n$ and $\left|E\left(\Gamma_{n}\right)\right|=12 n-3$, with structure as follows:

$$
\begin{aligned}
& V\left(\Gamma_{n}\right)=\left\{z_{i}, x_{i}, y_{i}: 1 \leq i \leq 2 n\right\}, \\
& E\left(\Gamma_{n}\right)=\left\{y_{i} y_{i+1}, x_{i} x_{i+1}, z_{i} z_{i+1}: 1 \leq i \leq 2 n-1\right\} \cup\left\{x_{i} y_{i}, x_{i} z_{i}, y_{i} z_{i}: 1 \leq i \leq 2 n\right\} .
\end{aligned}
$$

Figure 3 reveals general formation of $\Gamma_{n}$.

In Figure 4, we have shown the network $\Gamma_{2}$ and its contained cycles of orders $3,4, \ldots, 12$.

In the upcoming result, we show that the pancyclic network $\Gamma_{n}$ is S- $(a, 0)$-EAM total.

Theorem 14. For all positive integers $n$, the pancyclic network $\Gamma_{n}$ is $S-(a, 0)-E A M$ total having magic constant $18 n$.

Proof

(i) For $n=1$, the labelling $\left\{z_{1}, z_{2}, x_{1}, x_{2}, y_{1}, y_{2}: 3,5,2,4,1,6\right\}$ extends to an S-(18,0)-EAM total labelling of $\Gamma_{1}$ by Lemma 1.

(ii) For $n \geq 3$.

Define here a labelling $g: V\left(\Gamma_{n}\right) \longrightarrow\{1,2, \ldots, 6 n\}$ as

$$
\begin{aligned}
& g\left(x_{i}\right)= \begin{cases}3 i: i \equiv 1(\bmod 2), & 1 \leq i \leq 2 n-1, \\
3 i-1: i \equiv 0(\bmod 2), & 2 \leq i \leq 2 n,\end{cases} \\
& g\left(y_{i}\right)= \begin{cases}3 i-2: i \equiv 1(\bmod 2), & 1 \leq i \leq 2 n-1, \\
3 i: i \equiv 0(\bmod 2), & 2 \leq i \leq 2 n,\end{cases} \\
& g\left(z_{i}\right)= \begin{cases}3 i-1: i \equiv 1(\bmod 2), & 1 \leq i \leq 2 n-1, \\
3 i-2: i \equiv 0(\bmod 2), & 2 \leq i \leq 2 n .\end{cases}
\end{aligned}
$$

All edge sums generated by the above labelling scheme constitute a sequence of consecutive integers $3,4, \ldots, 12 n-1$. Therefore, by Lemma $1, g$ extends to an S- $(a, 0)$-EAM total labelling of $\Gamma_{n}$ having magic constant $a=18 n$.

Again from Theorem 1, we have a direct consequence as follows.

Theorem 15. For all positive integers $n$, the pancyclic network $\Gamma_{n}$ admits an $S-(6 n+4,2)-E A M$ total labelling. 


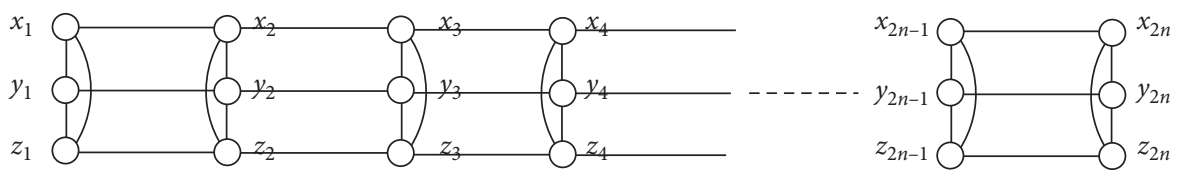

Figure 3: The general formation of the pancyclic network $\Gamma_{n}$.
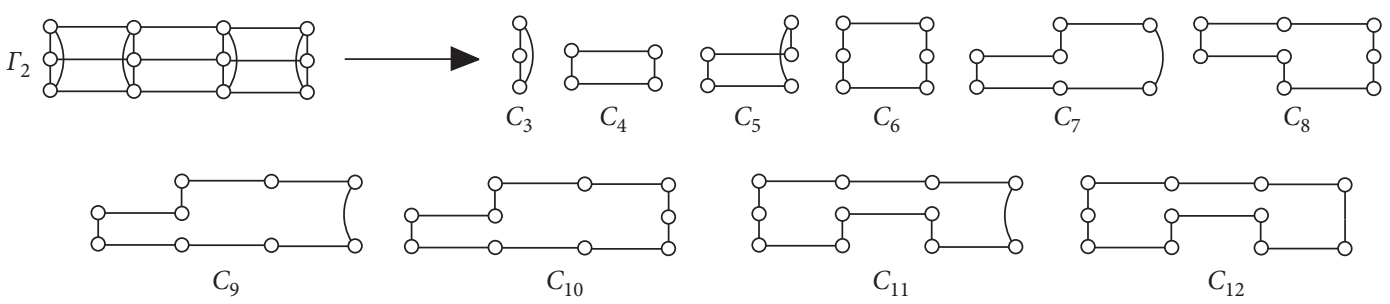

FIgure 4: The network $\Gamma_{2}$ having order 12 and its contained cycles $C_{3}, C_{4}, \ldots, C_{12}$.

2.4. S-(a,0)-EAM Total Labelling of Symmetric Lattice Networks

Definition 8. Consider a tripartite network $\mathbb{T}$ having vertexedge connections as follows:

$$
\begin{aligned}
& V(\mathbb{T})=\{x, y, z, u, v, w\}, \\
& E(\mathbb{T})=\{u v, v w, x y, y z, u y, w y, x v, z v, v y\} .
\end{aligned}
$$

In this section, we study the S- $(a, 0)$-EAM total labelling of symmetric lattice networks $\mathbb{L}_{n}^{1}, \mathbb{L}_{n}^{2}$, and $\mathbb{L}_{n}^{3}$. These symmetric lattices contain $n$ copies of the tripartite network $\mathbb{T}$.

Definition 9

(i) For $n=1, \mathbb{L}_{1}^{1} \cong \mathbb{T}$.

(ii) For $n \geq 2$.

The lattice network $\mathbb{L}_{n}^{1}$ is a network with order $6 n$ and size $12 n-3$ defined as follows:

$$
\begin{aligned}
V\left(\mathbb{L}_{n}^{1}\right)= & \left\{x_{i}, y_{i}, c_{i}: 1 \leq i \leq 2 n\right\} . \\
E\left(\mathbb{L}_{n}^{1}\right)= & \left\{c_{i} y_{i+1}, c_{i} x_{i+1}, c_{i} y_{i}, c_{i} x_{i}: 1 \leq i \leq 2 n-1, i \equiv 1(\bmod 2)\right\} \\
& \cup\left\{c_{i} c_{i+1}: 2 \leq i \leq 2 n-1\right\} \cup\left\{x_{i} x_{i+1}, y_{i} y_{i+1}: i \equiv 0(\bmod 2), 2 \leq i \leq 2(n-1)\right\}, \\
& \cup\left\{c_{i} y_{i-1}, c_{i} x_{i-1}, c_{i} y_{i}, c_{i} x_{i}: i \equiv 0(\bmod 2), 2 \leq i \leq 2 n\right\} .
\end{aligned}
$$

Figure 5 illustrates the general formation of the lattice network $\mathbb{L}_{n}^{1}, \forall n \in \mathbb{N}$.

Theorem 16. For all positive integers $n$, the lattice network $\mathbb{L}_{n}^{1}$ is $S$ - $(a, 0)-E A M$ total having magic constant $18 n$.

Proof

(i) For $n=1$ : the vertex labelling $\left\{x_{1}, c_{1}, y_{1}, x_{2}, c_{2}, y_{2}\right.$ : $3,1,2,5,6,4\}$ extends to an S- $(18,0)$-EAM total labelling of $\mathbb{L}_{1}^{1}$, by Lemma 1 .

(ii) For $n>1$.

We are defining a labelling $\varphi_{1}: V\left(\mathbb{L}_{n}^{1}\right) \longrightarrow\{1,2, \ldots, 6 n\}$ as follows:

$$
\begin{aligned}
\varphi_{1}\left(x_{i}\right) & = \begin{cases}3 i: i \equiv 1(\bmod 2), & 1 \leq i \leq 2 n-1, \\
3 i-1: i \equiv 0(\bmod 2), & 2 \leq i \leq 2 n,\end{cases} \\
\varphi_{1}\left(y_{i}\right) & = \begin{cases}3 i-1: i \equiv 1(\bmod 2), & 1 \leq i \leq 2 n-1, \\
3 i-2: i \equiv 0(\bmod 2), & 2 \leq i \leq 2 n,\end{cases} \\
\varphi_{1}\left(c_{i}\right) & = \begin{cases}3 i-2: i \equiv 1(\bmod 2), & 1 \leq i \leq 2 n-1, \\
3 i: i \equiv 0(\bmod 2), & 2 \leq i \leq 2 n .\end{cases}
\end{aligned}
$$

All edge sums generated by the above labelling scheme constitute a sequence of consecutive integers $3,4, \ldots$, $12 n-3$. So, by Lemma $1, \varphi_{1}$ extends to an S- $(a, 0)$-EAM total labelling of $\mathbb{L}_{n}^{1}$ admitting magic constant $18 n$.

\section{Definition 10}

(i) For $n=1, \mathbb{L}_{1}^{2} \cong \mathbb{T}$.

(ii) For $n \geq 2$. 


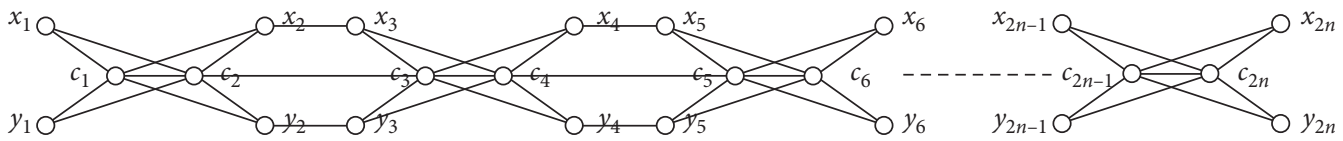

Figure 5: The general formation of the lattice network $\mathbb{L}_{n}^{1}, \forall n \in \mathbb{N}$.

The lattice network $\mathbb{L}_{n}^{2}$ having order $10 n-4$ and size $20 n-11$ is defined as follows:

$$
\begin{aligned}
V\left(\mathbb{L}_{n}^{2}\right) & =\left\{x_{i}, y_{i}: 1 \leq i \leq 2 n\right\} \cup\left\{c_{i}: 1 \leq i \leq 2(2 n-1)\right\} \cup\left\{u_{i}, v_{i}: 1 \leq i \leq n-1\right\} . \\
E\left(\mathbb{L}_{n}^{2}\right) & =\left\{c_{i} y_{i+2 / 2}, c_{i} x_{i+2 / 2}, c_{i} y_{i / 2}, c_{i} x_{i / 2}: 2 \leq i \leq 2(2 n-1), i \equiv 2(\bmod 4)\right\} \\
& \cup\left\{c_{i} y_{i+2 / 2}, c_{i} y_{i+3 / 2}, c_{i} x_{i+2 / 2}, c_{i} x_{i+3 / 2}: i \equiv 1(\bmod 4), 1 \leq i \leq 4 n-3\right\} \\
& \cup\left\{c_{i} c_{i+1}: 1 \leq i \leq 4 n-3, i \equiv 1(\bmod 4)\right\} \cup\left\{u_{i} v_{i}: 1 \leq i \leq n-1\right\} \\
& \cup\left\{x_{i} u_{i / 2}, y_{i} v_{i / 2}: 2 \leq i \leq 2(n-1), i \equiv 0(\bmod 2)\right\} \\
& \cup\left\{u_{i} x_{2 i+1}, v_{i} y_{2 i+1}, u_{i} c_{4 i-1}, v_{i} c_{4 i-1}, u_{i} c_{4 i}, v_{i} c_{4 i}: 1 \leq i \leq n-1\right\} \\
& \cup\left\{c_{i} c_{i+1}: i \equiv 0(\bmod 2), 2 \leq i \leq 4(n-1)\right\} .
\end{aligned}
$$

In Figure 6, we have presented the general formation of the lattice network $\mathbb{L}_{n}^{2}, \forall n \in \mathbb{N}$.

Theorem 17. For all positive integers $n$, the lattice network $\mathbb{L}_{n}^{2}$ is $S$ - $(a, 0)$-EAM total with magic constant $30 n-12$.

Proof (i) For $n=1$ : the vertex labelling $\left\{x_{1}, c_{1}, y_{1}, x_{2}, c_{2}, y_{2}\right.$ : $3,1,2,5,6,4\}$ extends to an S- $(18,0)$-EAM total labelling of $\mathbb{L}_{1}^{2}$, by Lemma 1 .

(ii) For $n>1$.

A labelling $\varphi_{2}: V\left(\mathbb{L}_{n}^{2}\right) \longrightarrow\{1,2, \ldots, 10 n-4\}$ is defined as follows:

$$
\begin{aligned}
& \varphi_{2}\left(x_{i}\right)= \begin{cases}5 i-2: i \equiv 1(\bmod 2), & 1 \leq i \leq 2 n-1, \\
5 i-5: i \equiv 0(\bmod 2), & 2 \leq i \leq 2 n,\end{cases} \\
& \varphi_{2}\left(y_{i}\right)= \begin{cases}5 i-3: i \equiv 1(\bmod 2), & 1 \leq i \leq 2 n-1, \\
5 i-6: i \equiv 0(\bmod 2), & 2 \leq i \leq 2 n,\end{cases} \\
& \varphi_{2}\left(c_{i}\right)= \begin{cases}\frac{1}{2}(5 i-3): 1 \leq i \leq 4 n-3, & i \equiv 1(\bmod 4), \\
\frac{1}{2}(5 i+2): 2 \leq i \leq 4 n-2, & i \equiv 2(\bmod 4), \\
\frac{1}{2}(5 i-1): 3 \leq i \leq 4 n-5, & i \equiv 3(\bmod 4), \\
\frac{1}{2}(5 i): 4 \leq i \leq 4(n-1), & i \equiv 0(\bmod 4),\end{cases} \\
& \varphi_{2}\left(u_{i}\right)=10 i-1, \quad 1 \leq i \leq n-1,
\end{aligned}
$$

All edge sums generated by the above labelling scheme constitute a sequence of consecutive integers $3,4, \ldots, 20 n-9$. So, by Lemma $1, \varphi_{2}$ extends to an S- $(a, 0)$-EAM total labelling of $\mathbb{\complement}_{n}^{2}$ having magic constant $30 n-12$.

\section{Definition 11}

(i) For $n=1, \mathbb{L}_{1}^{3} \cong \mathbb{T}$.

(ii) For $n \geq 2$. 


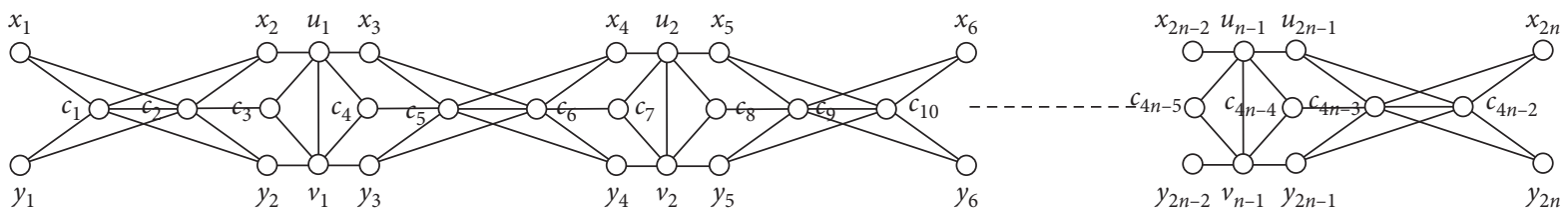

FIgURE 6: The general formation of the lattice network $\mathbb{L}_{n}^{2}, \forall n \in \mathbb{N}$.

The lattice network $\mathbb{L}_{n}^{3}$ having order $10 n-4$ and size $20 n-11$ is defined as follows:

$$
\begin{aligned}
V\left(\mathbb{L}_{n}^{3}\right)= & \left\{x_{i}, y_{i}: 1 \leq i \leq 2 n\right\} \cup\left\{c_{i}: 1 \leq i \leq 2(2 n-1)\right\} \cup\left\{u_{i}, v_{i}: 1 \leq i \leq n-1\right\}, \\
E\left(\mathbb{L}_{n}^{3}\right)= & \left\{c_{i} y_{i+2 / 2}, c_{i} x_{i+2 / 2}, c_{i} y_{i / 2}, c_{i} x_{i / 2}: 2 \leq i \leq 2(2 n-1), i \equiv 2(\bmod 4)\right\} \\
& \cup\left\{c_{i} y_{i+2 / 2}, c_{i} y_{i+3 / 2}, c_{i} x_{i+1 / 2}, c_{i} x_{i+3 / 2}: 1 \leq i \leq 4 n-3, i \equiv 1(\bmod 4)\right\} \\
& \cup\left\{x_{i} u_{i / 2}, y_{i} v_{i / 2}: i \equiv 0(\bmod 2), 2 \leq i \leq 2(n-1)\right\} \cup\left\{c_{i} c_{i+1}: 1 \leq i \leq 4 n-3\right\} \\
& \cup\left\{u_{i} x_{2 i+1}, v_{i} y_{2 i+1}, u_{i} c_{4 i-1}, v_{i} c_{4 i-1}, u_{i} c_{4 i}, v_{i} c_{4 i}: 1 \leq i \leq n-1\right\} .
\end{aligned}
$$

Figure 7 illustrates the general form of the lattice network $\mathbb{L}_{n}^{3}, \forall n \in \mathbb{N}$.

Theorem 18. For all positive integers $n$, the lattice network $\mathbb{L}_{n}^{3}$ is $S$ - $(a, 0)-E A M$ total with magic constant $30 n-12$.

Proof. For $\mathbb{L}_{n}^{3}$, the labelling design is similar as in Theorem 17.

\section{Illustration through Examples and Proposed Open Problems}

3.1. Examples. The S-(132,0)-EAM total labelling of $\left(C_{5} \circ K_{2,6}\right) \cup K_{1,5} \cup 2 K_{1}$ and S- $(163,0)$-EAM total labelling of $\left(C_{7} \circ K_{2,5}\right) \cup K_{1,7} \cup 3 K_{1}$ are presented, respectively, in Figures 8(a) and $8(\mathrm{~b})$. In Figure $8(\mathrm{a})$, the parameters are $\{n=5, m=6\}$ and $a=132$, while in Figure $8(\mathrm{~b})$, the parameters are $\{n=7, m=5\}$ and $a=163$. These are perfect according to our depiction of the magic constants in the proofs of Theorems 2 and 3.

Similarly, Figures 9 and 10 illustrate Theorems 4 and 5 and Theorems 6 and 7, respectively, for the values of the parameters given in each. The values of $a$ here are perfectly similar as depicted in our main findings.

Figures 11(a) and 11(b) illustrate Theorems 11 and 12, respectively, for $n=5$.

Figure 12 reveals an example of Theorem 15 corresponding to parameter $n=3$.

Figures $13-15$ refer to the illustration of S- $(a, 0)$-EAM total labelling of lattice networks $\mathbb{L}_{5}^{1}, \mathbb{L}_{4}^{2}$, and $\mathbb{L}_{4}^{3}$ (Theorems 16-18).

Due to facilitation of Lemma 1, edge labels are not needed to be provided in all of the above illustrative figures. As the edge sums constitute a sequence of +ve consecutive integers, assigning the remaining labels $\{q, q-1, \ldots, p+2, p+1\}$ to the edges in ascending or descending order will generate $\mathrm{S}$ - $\left(a^{\prime}, 2\right)$ or $(a, 0)$-EAM total labelling on that network, respectively, where $a$ (magic constant) and $a^{\prime}$ (minimum edge weight) attain some suitable values accordingly. More precisely, according to Lemma 1, this vertex labelling, consisting of consecutive integers, extends to an S- $(a, 0)$-EAM total labelling of the networks.

3.2. Open Problems. The open problems relevant to the findings (Theorems 2-7) of Section 2 are proposed as follows:

(i) For $2 \leq n \equiv 0(\bmod 2)$, obtain any $\mathrm{S}-(a, 0)$-EAM total labelling of the $\left(C_{n} \circ K_{2, m}\right) \cup K_{1, n} \cup(n-1 / 2) K_{1}$.

(ii) For $2 \leq n \equiv 0(\bmod 2)$, obtain any $\mathrm{S}-(a, 0)$-EAM total labelling of $\left(C_{n} \circ K_{2, m}\right) \cup n P_{2}$.

(iii) For $2 \leq n \equiv 0(\bmod 2)$, obtain any $\mathrm{S}$ - $(a, 0)$-EAM total labelling of $\left(C_{n} \circ K_{2, m}\right) \cup P_{n+1}$.

(iv) For $2 \leq n \equiv 0(\bmod 2)$, determine $\mathrm{S}$ - $(a, 0)$-EAM total labelling of $G_{1}, G_{2}, G_{3}, G_{4}, G_{5}$, and $G_{6}$ for any other magic constants (i.e., for any other value of $a$ ) than computed here.

(v) For $l, m$ and $n$ positive integers, determine any S- $(a, 0)$-EAM total labelling for the following networks:
(1) $\left(C_{n} \circ K_{2, m}\right) \cup K_{1, \ell}$
(2) $\left(C_{n} \circ K_{2, m}\right) \cup l P_{2}$
(3) $\left(C_{n} \circ K_{2, m}\right) \cup P_{l}$

Open problems related to Theorems 11 and 12 are given as follows:

(i) For $2 \leq n \equiv 0(\bmod 2)$, find any $\mathrm{S}$ - $(a, 0)$-EAM total labelling of $\mathrm{C}_{n} \circ \mathrm{H}_{1}$.

(ii) For $2 \leq n \equiv 0(\bmod 2)$, find any $\mathrm{S}$ - $(a, 0)$-EAM total labelling of $\mathrm{C}_{n} \circ \mathrm{H}_{2}$.

(iii) For $3 \leq n \equiv 1(\bmod 2)$, determine some $\mathrm{S}$ - $(a, 0)$ EAM total labelling of $\mathrm{C}_{n} \circ \mathrm{H}_{1}$ and $\mathrm{C}_{n} \circ \mathrm{H}_{2}$ with a different magic constant than obtained here, i.e., for any other value of $a$. 


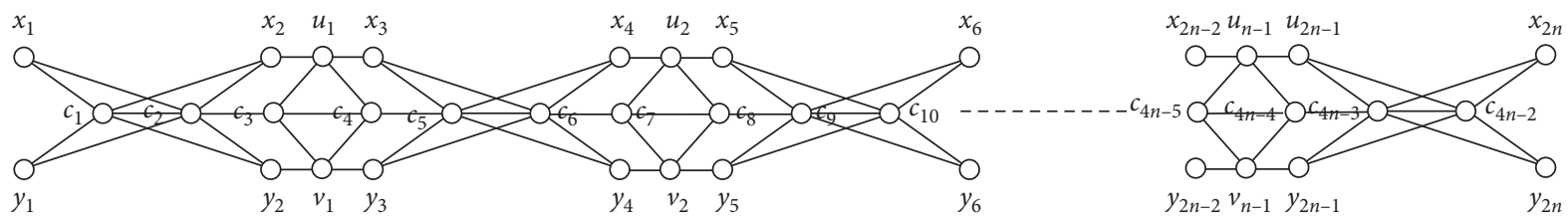

FIgURE 7: The general formation of the lattice network $\mathbb{L}_{n}^{3}, \forall n \in \mathbb{N}$.

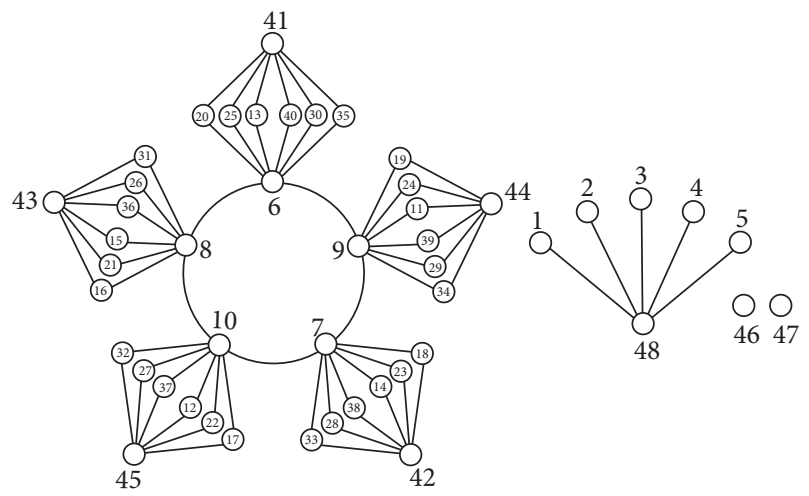

(a)

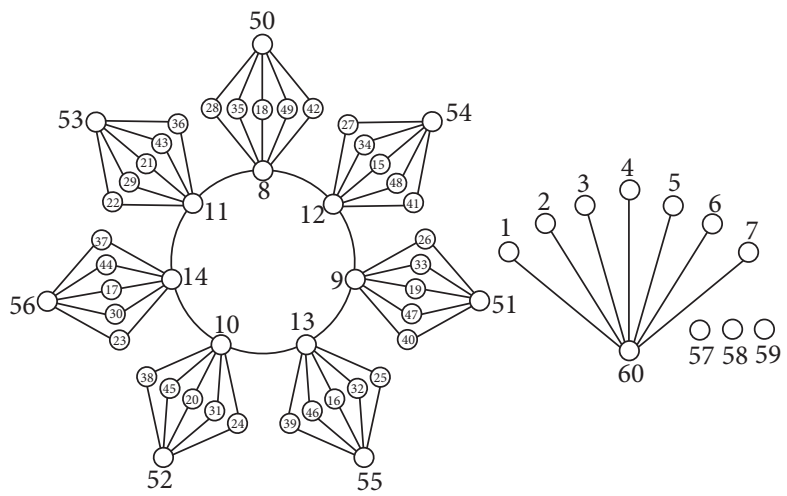

(b)

Figure 8: An S- $(132,0)$-EAM total labelling of $\left(C_{5} \circ K_{2,6}\right) \cup K_{1,5} \cup 2 K_{1}(\leftarrow A)$ and an S- $(163,0)$-EAM total labelling of $\left(C_{7} \circ K_{2,5}\right) \cup K_{1,7} \cup 3 K_{1}$ $(\leftarrow B)$.

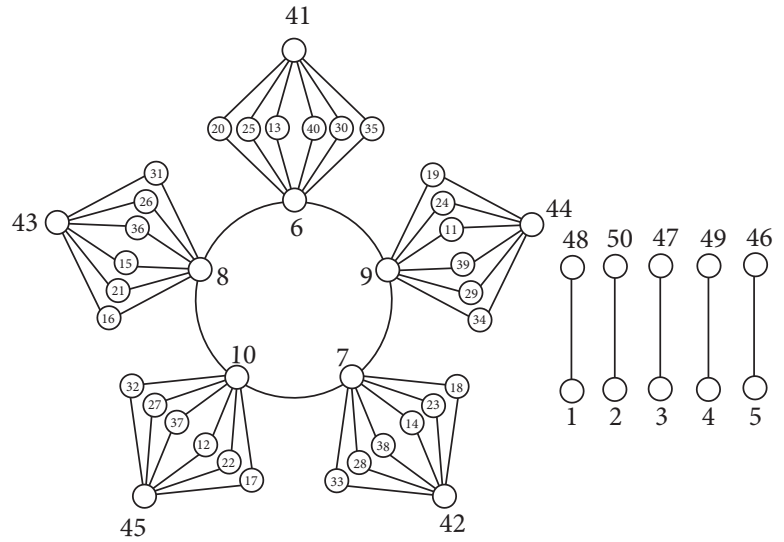

(a)

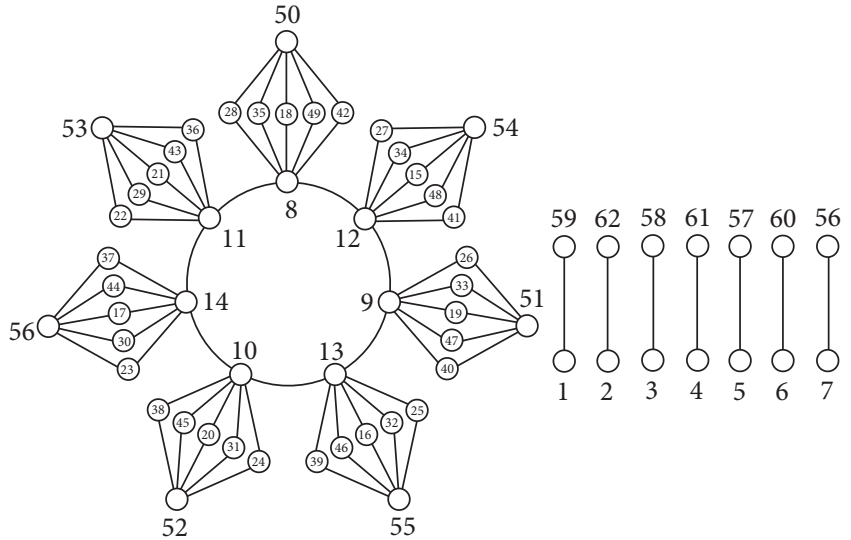

(b)

Figure 9: An S- $(134,0)$-EAM total labelling of $\left(C_{5} \circ K_{2,6}\right) \cup 5 P_{2}(\longrightarrow A)$ and an S- $(165,0)$-EAM total labelling of $\left(C_{7} \circ K_{2,5}\right) \cup 5 P_{2}(\longrightarrow B)$.

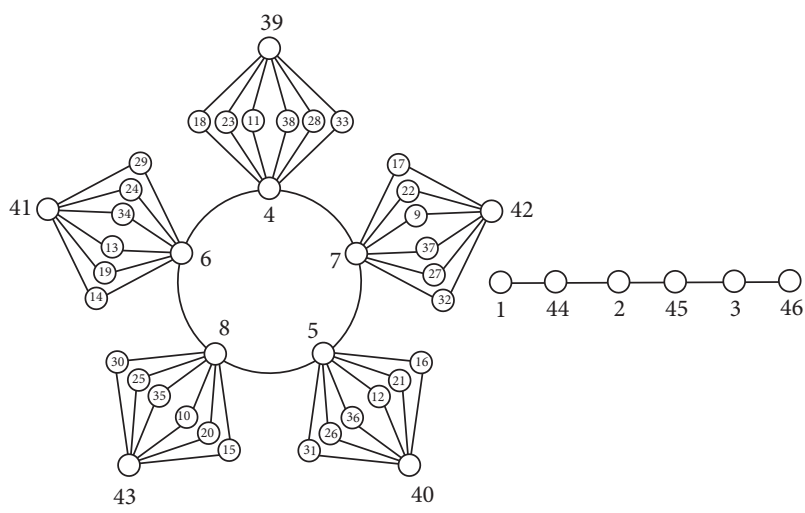

(a)

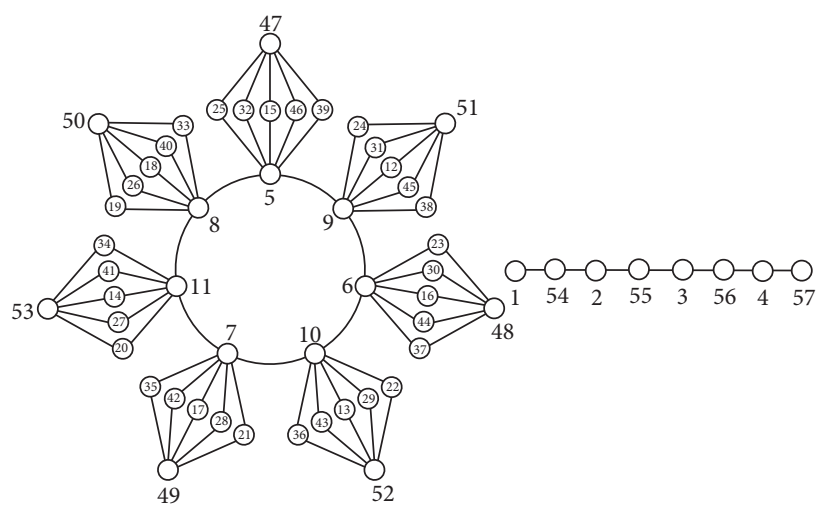

(b)

Figure 10: An S- $(126,0)$-EAM total labelling of $\left(C_{5} \circ K_{2,6}\right) \cup P_{6}(\longrightarrow A)$ and an S- $(154,0)$-EAM total labelling of $\left(C_{7} \circ K_{2,5}\right) \cup P_{8}(\longrightarrow B)$. 


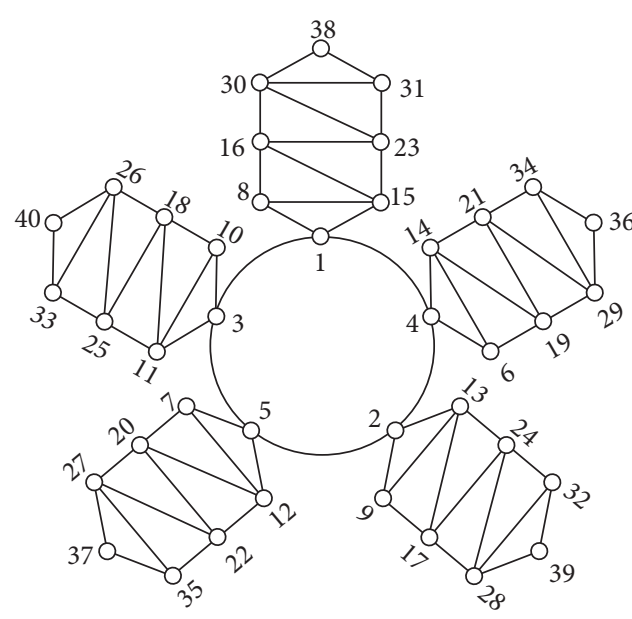

(a)

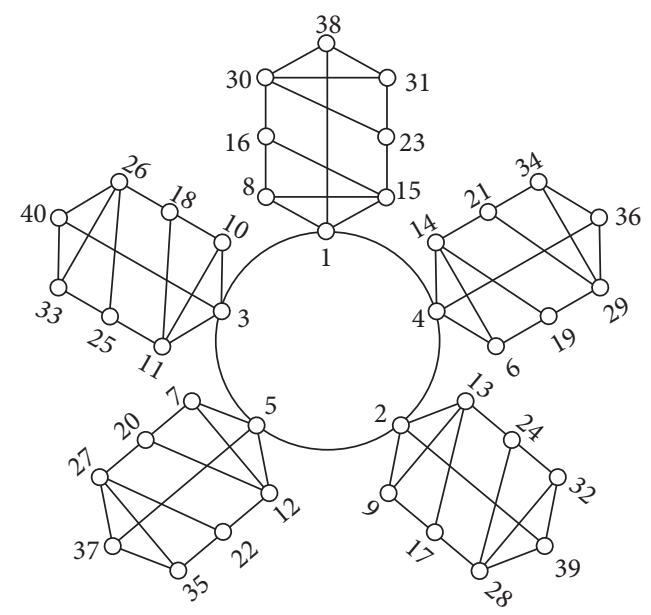

(b)

Figure 11: An S-(114,0)-EAM total labelling of $C_{5} \circ H_{1}(\longrightarrow A)$ and $C_{5} \circ H_{2}(\longrightarrow B)$.

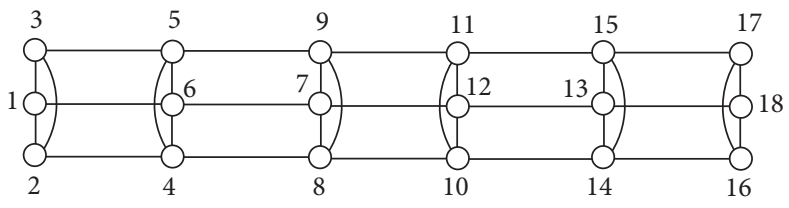

Figure 12: An S- $(54,0)$-EAM total labelling of $\Gamma_{3}$.

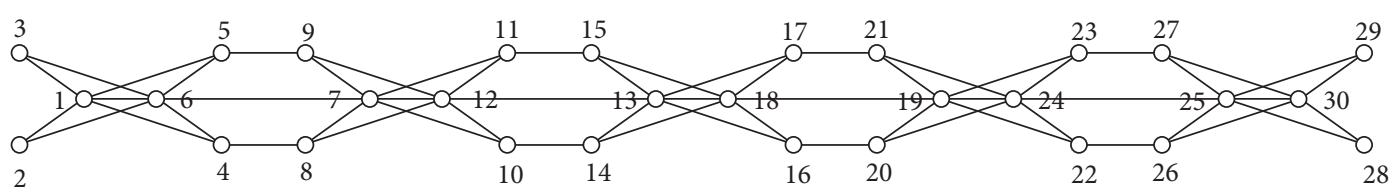

Figure 13: An S- $(90,0)$-EAM total labelling of the lattice network $\mathbb{L}_{5}^{1}$.

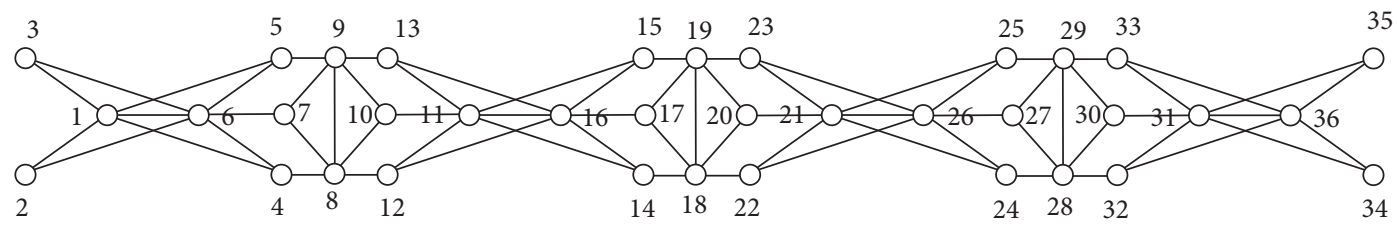

Figure 14: An S- $(108,0)$-EAM total labelling of the lattice network $\mathbb{L}_{4}^{2}$.

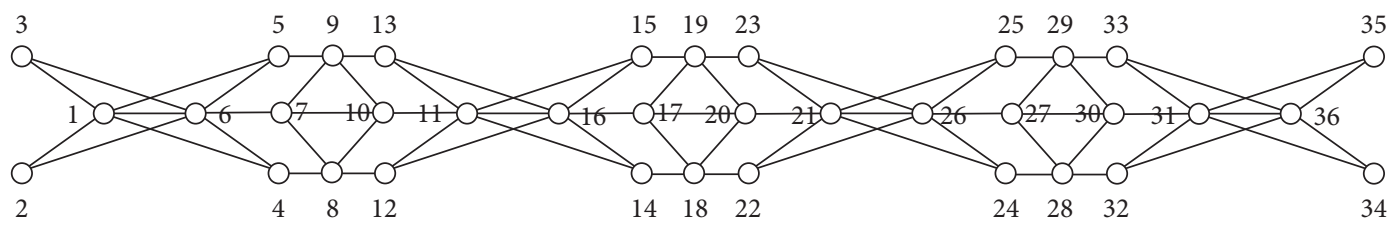

Figure 15: An S- $(108,0)$-EAM total labelling of the lattice network $\mathbb{L}_{4}^{3}$. 
TABLE 2: Synopsis of main theorems.

\begin{tabular}{lcccc}
\hline Network & Parameters & $a(d=0)$ & $a^{\prime}(d=2)$ & Planar V nonplanar \\
\hline$\left(C_{n} \circ K_{2, m}\right) \cup K_{1, n} \cup(n-1 / 2) K_{1}$ & $m \geq 2$, odd $n \geq 3$ & $8 n+3 m n+2$ & $6 n+m n+3$ & Planar \\
$\left(C_{n} \circ K_{2, m}\right) \cup n P_{2}$ & $m \geq 2$, odd $n \geq 3$ & $(1 / 2)(17 n+6 m n+3)$ & $(1 / 2)(13 n+2 m n+5)$ & Planar \\
$\left(C_{n} \circ K_{2, m}\right) \cup P_{n+1}$ & $m \geq 2$, odd $n \geq 3$ & $(1 / 2)(13 n+6 m n+7)$ & $(1 / 2)(9 n+2 m n+9)$ & Planar \\
$C_{n} \circ H_{1}$ and $C_{n} \circ H_{2}$ & $n$ is odd & $(1 / 2)(45 n+3)$ & $(1 / 2)(17 n+5)$ & Planar \\
$\Gamma_{n}$ & $\forall n \in \mathbb{N}$ & $18 n$ & $6 n+4$ & Planar \\
$\mathbb{L}_{n}^{1}$ & $\forall n \in \mathbb{N}$ & $18 n$ & $6 n+4$ & Nonplanar \\
$\mathbb{L}_{n}^{2}$ and $\mathbb{L}_{n}^{3}$ & $\forall n \in \mathbb{N}$ & $30 n-12$ & $10 n$ & Nonplanar \\
\hline
\end{tabular}

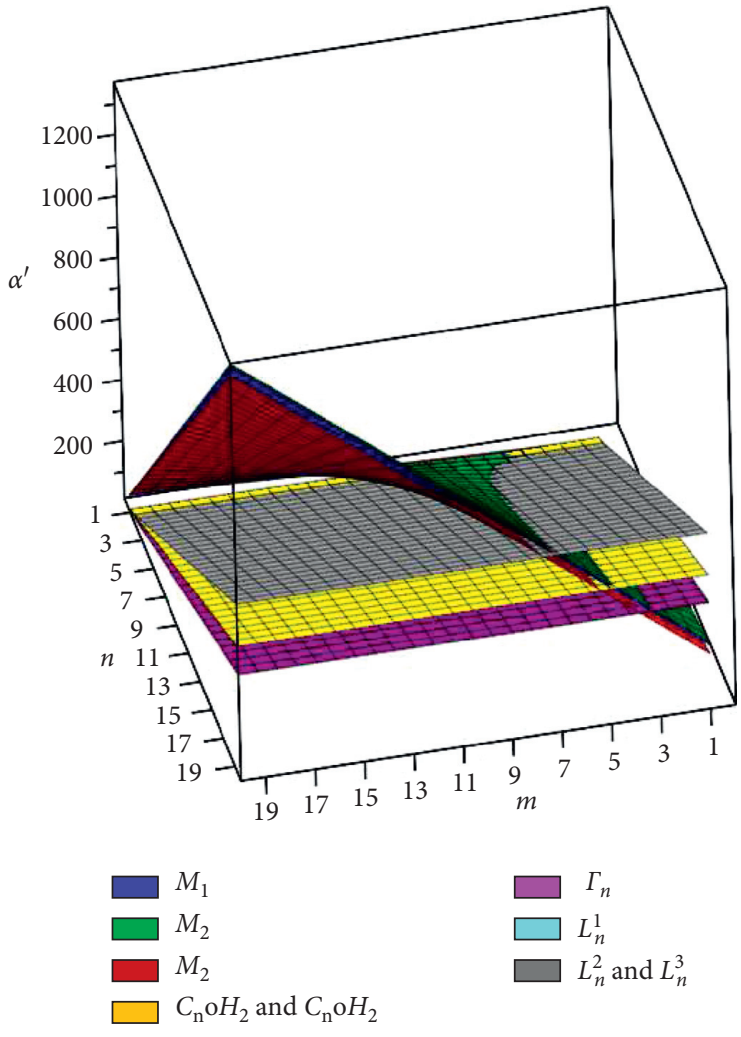

(a)

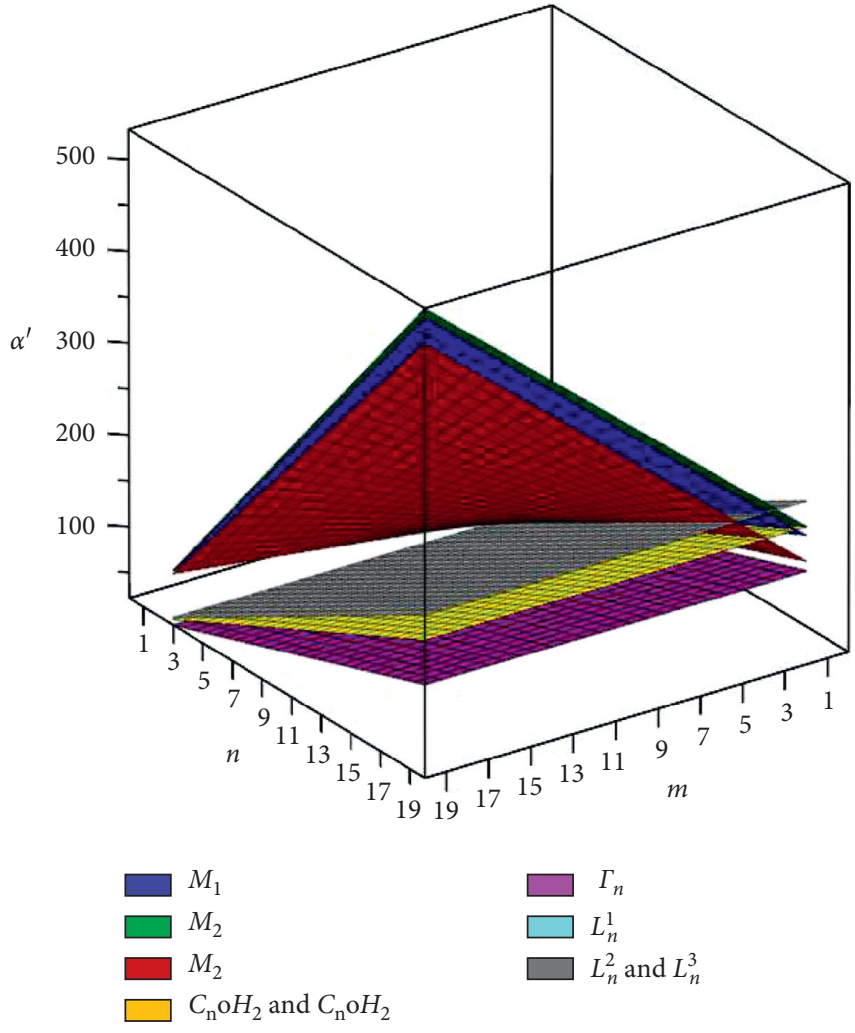

(b)

Figure 16: Comparison of the magic constants $(\longrightarrow A)$ and the minimum edge weights $(\longrightarrow B)$ of $M_{1}, M_{2}, M_{3}, C_{n} \circ H_{1}, C_{n} \circ H_{2}, \Gamma_{n}, \mathbb{L}_{n}^{1}, \mathbb{L}_{n}^{2}$, and $\mathbb{L}_{n}^{3}$, for $m \geq 2$ and $3 \leq n \equiv 1(\bmod 2)$.

\section{Synopsis and 3- $D$ Comparison of the Magic Constants and Minimum Edge Weights}

This section consists of the synopsis (Section 4.1) and 3-D graphical plots and comparison of the magic constants $(a)$ and minimum edge weights $\left(a^{\prime}\right)$ of our findings (Section 4.2).

4.1. Synopsis. Table 2 exhibits the computational results of our findings. The possible parameters for which we have determined S- $(a, 0)$ and S- $\left(a^{\prime}, 2\right)$-EAM total labellings are indicated through parameters column head.
4.2. Graphical Behavior of the Magic Constants and Minimum Edge Weights. Figure 16 shows the graphical comparison of the magic constants $(\longrightarrow A)$ and minimum edge weights $(\longrightarrow B)$, in 3-D, of the networks $M_{1} \cong\left(C_{n} \circ K_{2, m}\right) \cup K_{1, n} \cup(n-1 / 2) K_{1}$, $M_{2} \cong\left(C_{n} \circ K_{2, m}\right) \cup n P_{2}, M_{3} \cong\left(C_{n} \circ K_{2, m}\right) \cup P_{n+1}, C_{n} \circ H_{1}$, $C_{n} \circ H_{2}, \Gamma_{n}, \mathbb{L}_{n}^{1}, \mathbb{L}_{n}^{2}$, and $\mathbb{L}_{n}^{3}$, respectively. Moreover, Figure 16(a) shows that the most dominant layer is the one with green color. It interprets that among the magic constants of the networks discussed in this note, $\left(C_{n} \circ K_{2, m}\right) \cup n P_{2}$ attains highest values with the increase of the values of the parameters.

Figure 17 illustrates the relative 3-D comparison of the magic constants and minimum edge weights (corresponding to $d=2)$ of the networks $\left(C_{n} \circ K_{2, m}\right) \cup K_{1, n} \cup(n-1 / 2) K_{1}$, 

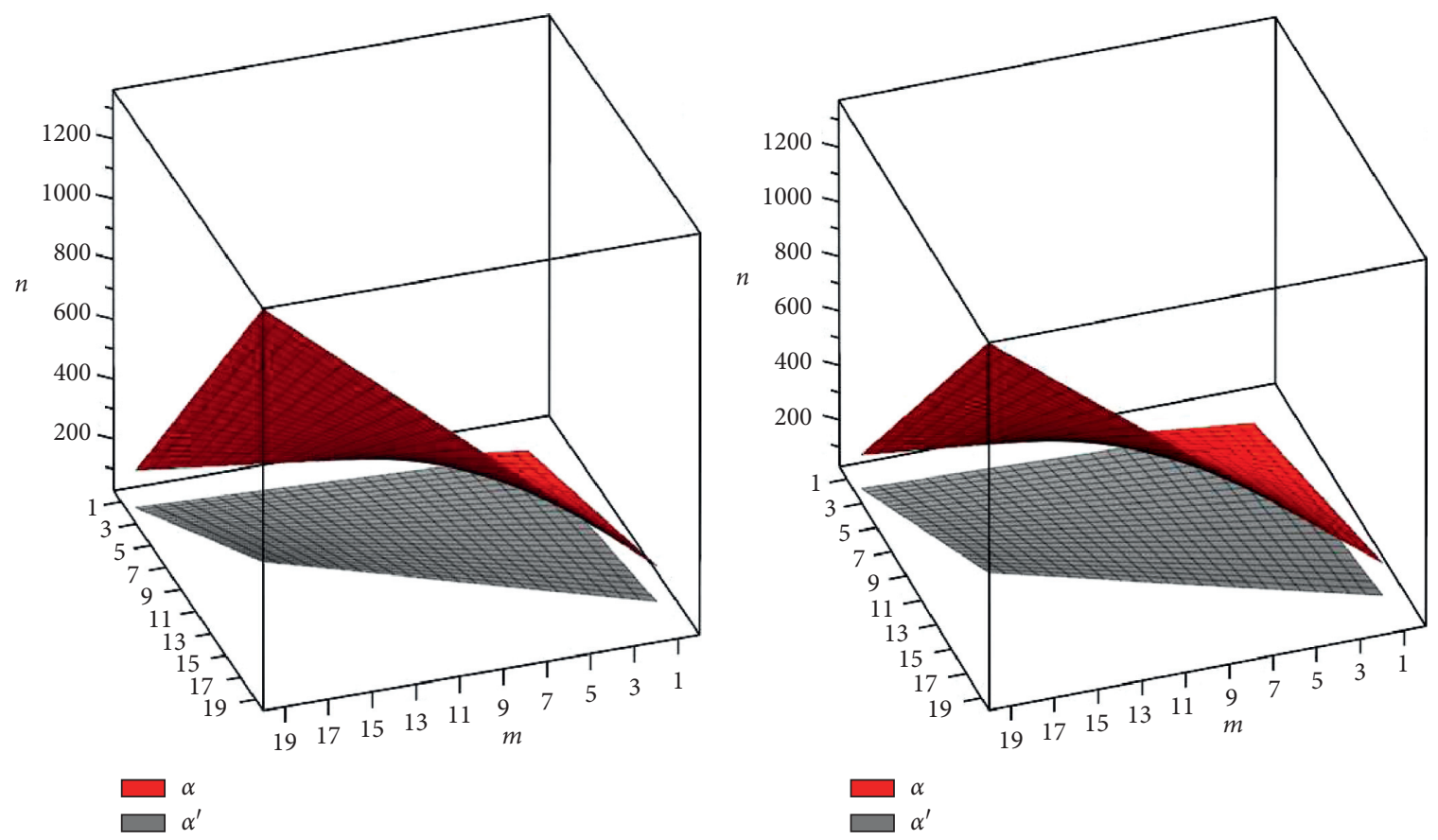

(a)

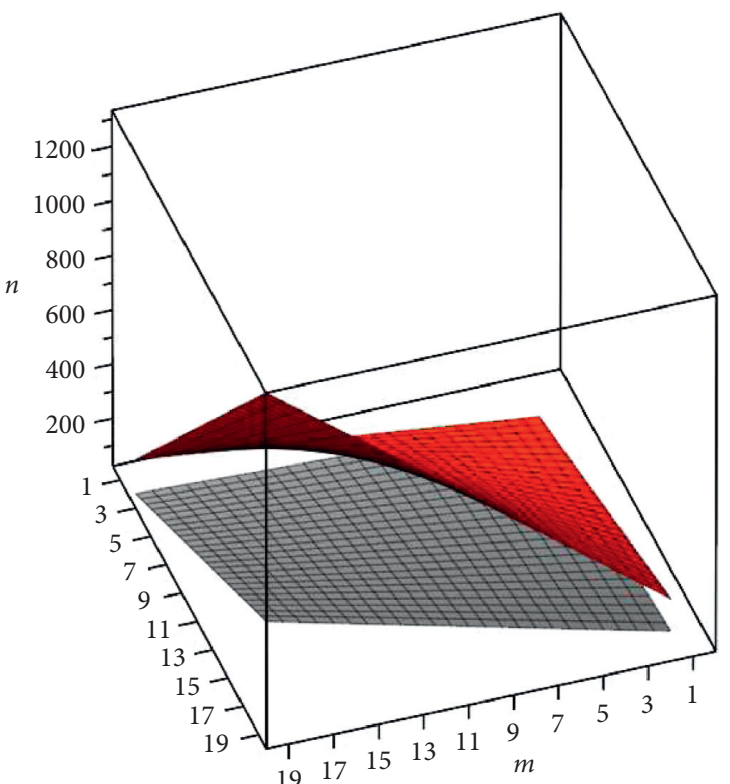

(b)

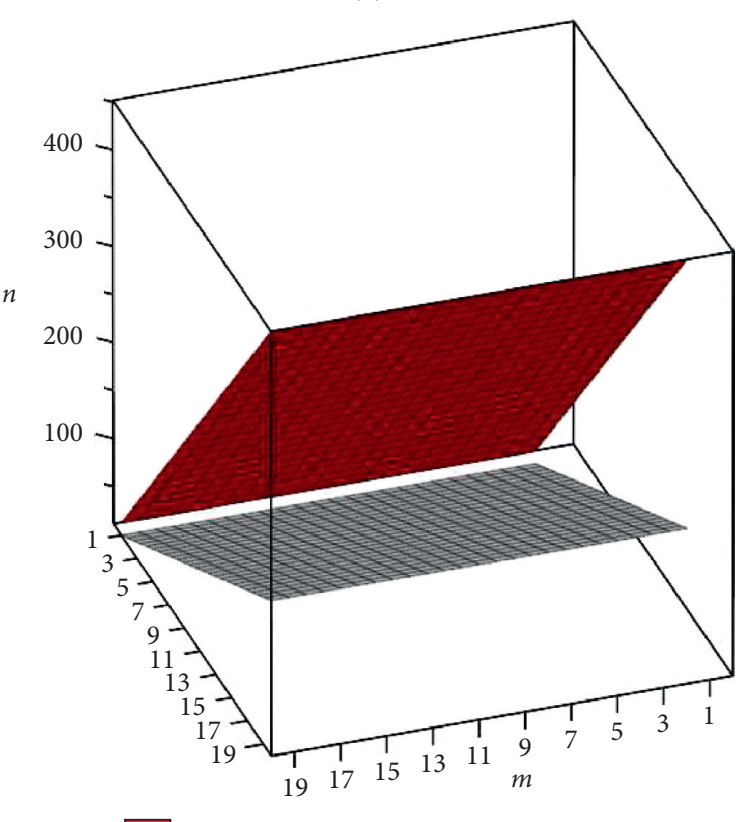

$\square \alpha$

$\square \alpha$

(c)

(d)

FIgUre 17: Continued. 


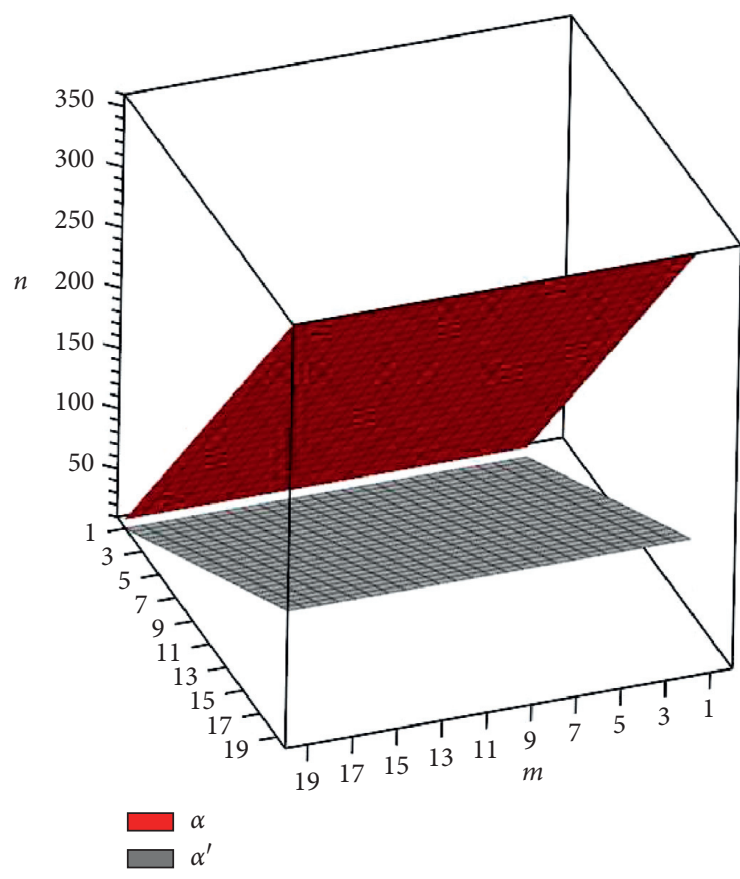

(e)

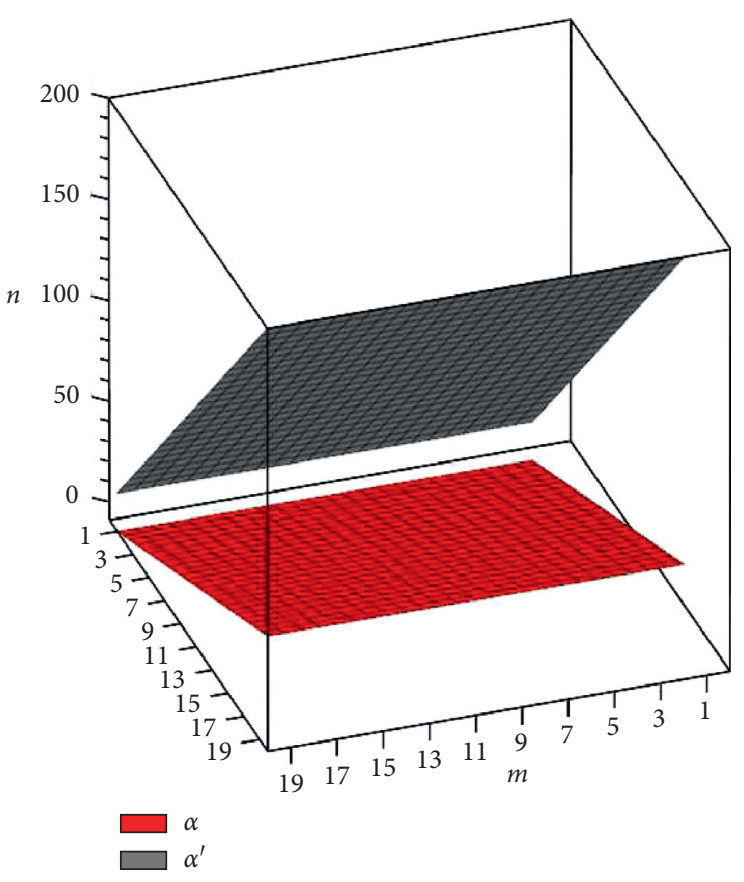

(f)

FiguRE 17: Simultaneous relative comparison of the magic constants and the minimum edge weights of $M_{1}(\longrightarrow A), M_{2}(\longrightarrow B), M_{3}(\longrightarrow C)$, $\left\{C_{n} \circ H_{1}, C_{n} \circ H_{2}\right\}(\longrightarrow D), \Gamma_{n}(\longrightarrow E),\left\{\mathbb{L}_{n}^{1}, \mathbb{L}_{n}^{2}, \mathbb{L}_{n}^{3}\right\}(\longrightarrow F)$, for $m \geq 2 \& 3 \leq n \equiv 1(\bmod 2)$

$\left(C_{n} \circ K_{2, m}\right) \cup n P_{2}, \quad\left(C_{n} \circ K_{2, m}\right) \cup P_{n+1}, C_{n} \circ H_{1}, C_{n} \circ H_{2}, \Gamma_{n}$, $\mathbb{L}_{n}^{1}, \mathbb{L}_{n}^{2}$ and $\mathbb{L}_{n}^{3}$, for different values of the parameters.

\section{Conclusion}

In the present article,

(i) We have designed S- $(a, 0)$-EAM total labelling of the rooted product of cycle $C_{n}$ and complete bipartite network $K_{2, m}$ taking its disjoint union with paths and stars. The findings are related to the open problem on $n K_{2, m}$ provided by Ngurah et al. in [48].

(ii) We have provided S- $(a, 0)$-EAM total labelling of rooted product of $C_{n}$ and pancyclic networks $H_{1}$ and $\mathrm{H}_{2}$.

(iii) We have extended the result provided in [29] by Baig et al. through exhibiting S- $(a, 0)$-EAM total labelling of pancyclic network $\Gamma_{n}$ involving chains of $C_{6}$.

(iv) We have exhibited S- $(a, 0)$-EAM total labelling of symmetrically designed lattice networks $\mathbb{L}_{n}^{1}$, $\mathbb{L}_{n}^{2}$, and $\mathbb{L}_{n}^{3}$.

(v) We have illustrated our findings through 3-D graphical comparison.

(vi) For further working in this field, several research problems have also been opened.

(vii) The obtained schemes are now all set to serve as test ready labellings for programmers, networking professionals, and engineers to avail them where they find these appropriate.

\section{Data Availability}

The data used to support the findings of this study are included within this article. However, the reader may contact the corresponding author for more details on the data.

\section{Conflicts of Interest}

The authors declare that they have no conflicts of interest.

\section{References}

[1] Z. Shao, " $L(2,1)$-labeling of the strong product of paths and cycles," The Scientific Wolrd Journal, vol. 2014, Article ID 741932, 2014.

[2] T.-M. Wang and C.-C. Hsiao, "On anti-magic labeling for graph products," Discrete Mathematics, vol. 308, no. 16, pp. 3624-3633, 2008.

[3] D. B. West, Introduction to Graph Theory, Prentice-Hall, Upper Saddle River, NJ, USA, 2001.

[4] J. Sedl, "Problem 27 in the theory of graphs and its applications," in Proceedings of the Symposium, pp. 163-167, Smolenice, Slovakia, 1963.

[5] N. Hartsfield and G. Ringel, Pearls in Graph Theory, Academia Press, New York, NY, USA, 1990.

[6] A. Kotzig and A. Rosa, "Magic valuations of finite graphs," Canadian Mathematical Bulletin, vol. 13, no. 4, pp. 451-461, 1970.

[7] G. Ringel and A. S. Lldo, "Another tree conjecture," Bull ICA, vol. 18, pp. 83-85, 1996.

[8] H. Enomoto, A. Llado, T. Nakamigawa, and G. Ringel, "Super edge-magic graphs," SUT Journal of Mathematics, vol. 34, pp. 105-109, 1998. 
[9] R. Simanjuntak, F. Bertault, and M. Miller, "Two new (a,d)- EAT graph labelings," in Proceedings of the Eleventh Austrailian Workshop of Combinatorial Algorithm, pp. 179-189, Ballarat, Australia, 2000.

[10] S. M. Lee and Q. X. Shan, All Trees with at Most 17 Vertices Are Super Edge-Magic, University Southern Illinois., Carbondale, Australia, 2002.

[11] M. Javaid, A. A. Bhatti, and M. K. Aslam, "Super (a,d)-edge antimagic total labeling of a subclass of trees," AKCE International Journal of Graphs and Combinatorics, vol. 14, no. 2, pp. 158-164, 2017.

[12] M. Javaid, "On super edge-antimagic total labeling of subdivided stars," Discussiones Mathematicae Graph Theory, vol. 34, no. 4, pp. 691-705, 2014.

[13] M. Javaid and A. A. Bhatti, "On super edge- antimagic total labeling of subdivided stars," Ars Combinatoria, vol. 105, pp. 503-512, 2012.

[14] A. Raheem, A. Q. Baig, and M. Javaid, "On (a, d) -EAT labeling of subdivision of $\mathrm{K} 1, \mathrm{r}$," Journal of Information and Optimization Sciences, vol. 39, no. 3, pp. 643-650, 2018.

[15] A. N. M. Salman, A. A. G. Ngurah, and N. Izzati, "On super edge- magic total labeling of a subdivision of a star," Utiltas Mathematica, vol. 81, pp. 275-284, 2010.

[16] M. Javaid and A. A. Bhatti, "On super edge- antimagic total labeling of generalized extended -trees," AKCE International Journal of Graphs and Combinatorics, vol. 11, pp. 115-126, 2014.

[17] M. Javaid, A. A. Bhatti, and M. Hussain, "On edge- antimagic total labeling of extended -trees," Utilitas Math, vol. 87, pp. 293-303, 2012.

[18] M. Javaid, M. Hussain, K. Ali, and K. H. Dar, "Super edgemagic total labeling on -trees," Utilitas Math, vol. 86, pp. 183-191, 2011.

[19] M. Hussain and E. T. Boskoro, "Slamin, on super edge magic total labeling of banana trees," Utiltas Mathematica, vol. 79, pp. 243-251, 2009.

[20] K. A. Sugeng, M. Miller, and M. Baa, "edge- antimagic total labelings of caterpillars," Lecture Notes in Computer Science, vol. 3330, Article ID 169aAS180, 2005.

[21] M. Javaid, A. A. Bhatti, and M. Hussain, "On super edgeantimagic total labeling of subdivided caterpillar," Utilitas Math, vol. 98, pp. 227-241, 2015.

[22] R. M. Figueroa-Centeno, R. Ichishima, and F. A. MuntanerBatle, "On super edge- magic graph," Ars Combinatoria, vol. 64, pp. 81-95, 2002.

[23] A. A. Bhatti, A. Nisar, and M. Kanwal, "Radio number of wheel like graphs," International Journal on Applications of Graph Theory In Wireless Ad Hoc Networks And Sensor Networks, vol. 3, no. 4, pp. 39-57, 2011.

[24] J. A. Gallian, "A dynamic survey of graph labeling," The Electronic Journal of Combinatorics, 2019.

[25] R. Khennoufa and O. Togni, "The radio antipodal and radio numbers of the hypercube," Ars Combinatoria, vol. 102, pp. 447-461, 2011.

[26] R. Figueroa-Centeno, R. Ichishima, and F. Muntaner-Batle, "On edge-magic labelings of certain disjoint unions of graphs,” Australas. J. Combin.vol. 32, pp. 225-242, 2005.

[27] R. M. Figueroa-Centeno, R. Ichishima, F. A. Muntaner-Batle, and A. Oshima, "A magical approach to some labeling conjectures," Discussiones Mathematicae Graph Theory, vol. 31, no. 1, pp. 79-113, 2011.

[28] R. M. Figueroa-Centeno, R. Ichishima, and F. A. MuntanerBatle, "The place of super edge-magic labelings among other classes of labelings," Discrete Mathematics, vol. 231, no. 1-3, pp. 153-168, 2001.
[29] A. Q. Baig, H. U. Afzal, M. Imran, and I. Javaid, "Super edgemagic labeling of volvox and pancyclic graphs," Util. Math.vol. 93, pp. 49-56, 2014.

[30] J.-B. Liu, M. K. Aslam, M. Javaid, and A. Raheem, "Computing edge-weight bounds of antimagic labeling on a class of trees," IEEE Access, vol. 7, pp. 93375-93386, 2019.

[31] A. Ahmad, M. F. Nadeem, and A. Gupta, "On super edge- magic deficiency of certain Toeplitz graphs," Hacettepe Journal of Mathematics and Statistics, vol. 47, no. 3, pp. 1-7, 2018.

[32] A. Ahmad, K. Ali, M. Bača, P. Kovář, and A. SemaničováFeňovčíková, "Vertex-antimagic labelings of regular graphs," Acta Mathematica Sinica, English Series, vol. 28, no. 9, pp. 1865-1874, 2012.

[33] M. Bača and M. K. Siddiqui, "Construction of tree from smaller graceful trees," Utilitas Mathematica, vol. 99, no. 1, pp. 175-186, 2016.

[34] A. Ahmad and F. A. Muntaner-Batle, "On super edge magic deficiency of unicyclic graphs," Utilitas Math, vol. 98, pp. 379-386, 2015.

[35] A. Ahmad, A. Q. Baig, and M. Imran, "On super edge-magicness of graphs,” Utilitas Math, vol. 89, pp. 373-380, 2012.

[36] P. R. L. Pushpam and A. Saibulla, "Super edge- antimagic total labeling of some classes of graphs," SUT Journal of Mathematics, vol. 48, no. 1, pp. 1-12, 2012.

[37] N. L. Prasanna, K. Sravanthi, and N. Sudhakar, "Applications of graph labeling in major areas of computer science," International Journal of Research in Computer and Communication Technology, vol. 3, pp. 819-823, 2014.

[38] N. L. Prasanna, "Applications of graph labeling in communication networks," Oriental Journal of Computer Science and Technology, vol. 7, pp. 893-897, 2014.

[39] M. Andreas, Wireless Communications, Wiley-IEEE, New York, NY, USA, 2005.

[40] R. Wakefield, Radio Broadcasting, W2UC, Union College, Schenectady, NY, USA, 1959.

[41] Z. Zhou, S. Das, and H. Gupta, "Connected K-coverage problem in sensor networks," in Proceedings. 13th International Conference on Computer Communications and Networks, pp. 373-378, IEEE Cat. No.04EX969), Chicago, IL, USA, 2004.

[42] M. S. Vinutha and P. Arathi, "Applications of graph coloring and labeling in computer science," International Journal on Future Revolution in Computer Science and Communication Engineering, vol. 3, pp. 14-15, 2017.

[43] I. Katzela and M. Naghshineh, "Channel assignment schemes for cellular mobile telecommunication systems: a comprehensive survey," IEEE Personal Communications, vol. 3, no. 3, pp. 10-31, 1996.

[44] A. Krishnaa, "On antimagic labellings of some cycle related graphs," Journal of Discrete Mathematical Sciences and Cryptography, vol. 15, pp. 225-235, 2012.

[45] A. Krishnaa, "Formulas and algorithms of antimagic labelings of Some helm related graphs," Journal of Discrete Mathematical Sciences and Cryptography, vol. 19, no. 2, pp. 435-445, 2016.

[46] A. Krishnaa, "Some applications of labelled graphs," International Journal of Mathematics Trends and Technology, vol. 37, no. 3, pp. 19-23, 2016.

[47] J. P. Desai, "A graph theoretic approach for modeling mobile robot team formations," Journal of Robotic Systems, vol. 19, no. 11, pp. $511-525,2002$.

[48] A. A. G. Ngurah, E. T. Baskoro, R. Simanjuntak, and S. Uttunggadewa, "On the super edge-magic strength and deficiency of graphs," Kyoto CGGT, vol. 4535, pp. 144-154, 2008. 